\title{
MaMAPK3-MaICE1-MaPOD P7 pathway, a positive regulator of cold tolerance in banana
}

Jie Gao ${ }^{1,2 \dagger}$, Tongxin Dou ${ }^{1 \dagger}$, Weidi He ${ }^{1 \dagger}$, Ou Sheng ${ }^{1}$, Fangcheng $\mathrm{Bi}^{1}$, Guiming Deng ${ }^{1}$, Huijun Gao ${ }^{1}$, Tao Dong ${ }^{1}$, Chunyu $\mathrm{Li}^{1}$, Sheng Zhang ${ }^{3}$, Ganjun $\mathrm{Yi}^{1}$, Chunhua $\mathrm{Hu}^{1 *}$ and Qiaosong Yang ${ }^{1 *}$ (D)

\begin{abstract}
Background: Banana is a tropical fruit with a high economic impact worldwide. Cold stress greatly affects the development and production of banana.

Results: In the present study, we investigated the functions of MaMAPK3 and MalCE1 involved in cold tolerance of banana. The effect of RNAi of MaMAPK3 on Dajiao (Musa spp. 'Dajiao'; ABB Group) cold tolerance was evaluated. The leaves of the MaMAPK3 RNAi transgenic plants showed wilting and severe necrotic symptoms, while the widetype (WT) plants remained normal after cold exposure. RNAi of MAMAPK3 significantly changed the expressions of the cold-responsive genes, and the oxidoreductase activity was significantly changed in WT plants, while no changes in transgenic plants were observed. MalCE1 interacted with MaMAPK3, and the expression level of MalCE1 was significantly decreased in MaMAPK3 RNAi transgenic plants. Over-expression of MalCE1 in Cavendish banana (Musa spp. AAA group) indicated that the cold resistance of transgenic plants was superior to that of the WT plants. The POD P7 gene was significantly up-regulated in MalCE1-overexpressing transgenic plants compared with WT plants, and the POD P7 was proved to interact with MalCE1.

Conclusions: Taken together, our work provided new and solid evidence that MaMAPK3-MaICE1-MaPOD P7 pathway positively improved the cold tolerance in monocotyledon banana, shedding light on molecular breeding for the cold-tolerant banana or other agricultural species.
\end{abstract}

Keywords: MaMAPK3, MalCE1, MaPOD P7, Antioxidant capacity, Cold tolerance

\footnotetext{
* Correspondence: huchunhua007@126.com; soyang@hotmail.com

${ }^{\dagger} \mathrm{Jie}$ Gao, Tongxin Dou and Weidi He contributed equally to this work.

'Institute of Fruit Tree Research, Guangdong Academy of Agricultural

Sciences; Key Laboratory of South Subtropical Fruit Biology and Genetic

Resource Utilization, Ministry of Agriculture and Rural Affairs, Guangdong Key

Laboratory of Tropical and Subtropical Fruit Tree Research, Guangzhou

510640, China

Full list of author information is available at the end of the article
}

(c) The Author(s). 2021 Open Access This article is licensed under a Creative Commons Attribution 4.0 International License, which permits use, sharing, adaptation, distribution and reproduction in any medium or format, as long as you give appropriate credit to the original author(s) and the source, provide a link to the Creative Commons licence, and indicate if changes were made. The images or other third party material in this article are included in the article's Creative Commons licence, unless indicated otherwise in a credit line to the material. If material is not included in the article's Creative Commons licence and your intended use is not permitted by statutory regulation or exceeds the permitted use, you will need to obtain permission directly from the copyright holder. To view a copy of this licence, visit http://creativecommons.org/licenses/by/4.0/. The Creative Commons Public Domain Dedication waiver (http://creativecommons.org/publicdomain/zero/1.0/) applies to the data made available in this article, unless otherwise stated in a credit line to the data. 


\section{Background}

Bananas (Musa spp.), including dessert and cooking types, are large herbaceous plants that are perennial but monocarpic [1]. The Musa originated in Southeast Asia and the Western Pacific region, and the domestication process started about 7000 years ago [2]. It involves hybridizations between diverse species and subspecies fostered by human migrations [3], selection of seedless diploid and triploid, and parthenocarpic hybrids widely dispersed by vegetative propagation. Half of the current production relies on some clones derived from a single triploid genotype (Cavendish) [4]. As a staple food and fruit for millions of people, bananas are one of the major export commodities of several developing countries, representing the largest international trade in fruits [5-7].

As an important and restricting factor, temperature determines the development and output of banana, since its growth and production will be irreversibly affected when the temperature is lower than $12^{\circ} \mathrm{C}$ [8]. Like many important crops originated in the tropics and subtropics [9], Musa spp. appears to lack the mechanism of cold acclimatization, while different cultivars exhibit various cold resistances, in which 'Dajiao' (Musa spp. 'Dajiao'; ABB Group) has a stronger cold tolerance compared with Cavendish Banana (Musa spp. Cavendish; AAA Group). It is fundamentally necessary to understand the regulatory mechanisms of cold-signaling pathways in banana to improve the cold tolerance in banana cultivars. Due to the highly heterozygous genotype and complex genetic background in molecular biology studies, such as gene function verification related to important traits, the study on cold resistance of bananas has been a challenge for a long time $[1,2]$.

MAPK cascades have been confirmed in response to many abiotic factors [10-15]. Plant plasma membrane receptors perceive threats and activate mitogen-activated protein kinase kinase kinase (MAPKKK). MAPKKK then phosphorylates mitogen-activated protein kinase kinase (MAPKK), and subsequently, mitogen-activated protein kinase (MAPK) will be phosphorylated by the activated MAPKK [16]. MAPKKK1-MAPKK2-MAPK4/6 cascade has been confirmed to participate in the positive regulation of cold treatment $[15,17]$. Over-expression of MAPK K2 in Arabidopsis thaliana results in an enhanced cold tolerance, while mapkk2 mutant exhibits an increased freezing sensitivity [15]. MAPK4/6 is phosphorylated by the activated MAPKK2 for the regulation of downstream components to adapt to the cold stress $[15,18]$. A previous report has shown that MYB15 is a phosphorylated substrate of MAPK6, and it is involved in cold resistance [19]. However, a conflict finding has also been reported last year that MPK6 is not the downstream element of MAPKK2, and the MAPKKK1-MAPKK2-MAPK4 pathway constitutively inhibits the activities of MAPK3 and
MAPK6 to strengthen the cold resistance [20]. Another cascade, MAPKK4/5-MAPK3/6, can also respond to cold stress. However, there is a controversy about the function of MAPK3/6 in cold resistance. In tobacco and rice, overexpression of MAPK3 gene confers cold tolerance by stimulating the expressions of COR genes [21, 22]. In Arabidopsis thaliana, the stability and transcriptional activity of ICE1, a basic-helix-loop-helix transcription factor (bHLH TF) regulating the expressions of $C B F$ genes, is reduced by the phosphorylation of MAPK3 and MAPK6, resulting in an impaired cold resistance [20, 23]. Collectively, MAPKKK1-MAPKK2-MAPK4/6 and MAPKK4/5MAPK3/6 cascades are activated upon cold stress, while their underlying regulatory mechanisms seem to be diverse in different species.

Some bHLH family members have been found to play roles in downstream of MAPKs. Their stability and transcriptional activity are impaired by the phosphorylation of MAPK cascades [20, 21, 23]. In Arabidopsis thaliana, MAPKK4/5-MAPK3/6 cascade can negatively modulate the cold response by regulating the stability of ICE1 protein, while the MAPKKK1-MAPKK2-MAPK4 cascade constitutively inhibits the activities of MAPK3 and MAPK6, playing a vigorous role in the cold response [20, 23]. In rice, OsbHLH002/OsICE1 is phosphorylated by OsMAPK3, and the interaction inhibits the ubiquitination of OsbHLH002/OsICE1 to promote the expression of OsTPP1 and improve the cold resistance [21]. A total of 162,167, and 152 bHLH genes have been identified in Arabidopsis thaliana, rice [24], and tomato [25], respectively. Moreover, bHLH proteins in plants participate in a wide variety of biological activities, including flowering [26], trichome and root hair differentiation [27, 28], flavonoid biosynthesis [29], chloroplast growth [30], photomorphogenesis [31], isoquinoline alkaloid [32] and anthocyanin biosynthesis [33]. A great deal of evidence has shown that bHLH transcript factors (TFs) also have fundamental functions in response to cold exposure in plants. For example, the bHLH TFs ICE1 and ICE2 of Arabidopsis thaliana and SIICE1a of tomato have been shown to participate in the response to cold stress $[34,35]$. Because of the huge damage induced by cold exposure, the functions of bHLH TFs in cold resistance are always a hot research topic in fruit science. In apple, MdCIbHLH1 protein has been found to bind to the promoter of $M d C B F 2$ and positively regulate the cold resistance in different species [36]. In Poncirus trifoliate, a bHLH TF named PtrbHLH modulates peroxidase-mediated scavenging of hydrogen peroxide, leading to enhanced cold resistance in transgenic tobacco and lemon [37], and PtrICE1 from trifoliate orange positively regulates cold resistance of tobacco and lemon by regulating polyamine contents via interplay with arginine decarboxylase [38]. In grape, VabHLH1 is 
cloned from a cold-tolerant Chinese wild Vitis amurensis, conferring cold tolerance in Arabidopsis thaliana [39].

Our laboratory has been undertaking a study on molecular mechanisms of cold tolerance in banana since 2012 using a variety of biology approaches, including transcriptomics, proteomics, phosphoproteomics, and genetic transformation methods [40-44]. We have shown that MAPK cascades, ICE1 signaling pathway and antioxidation mechanism play critical roles in cold resistance of banana. To provide more valuable insights into the correlation between MAPK cascades and ICE1 signaling pathway, we investigated the roles of MaMAPK3 and MaICE1 in low-temperature signaling in banana. In the present study, we analyzed the differences of phenotype, gene expression, potential interactions, and antioxidant ability in wide-type (WT), two individual MaMAPK3 RNAi transgenic 'Dajiao' lines and two individual MaICE-overexpressing transgenic Cavendish banana lines. Our data showed that MaMAPK3MaICE1-MaPOD P7 pathway appeared to positively regulate the cold resistance in banana.

\section{Results \\ RNAi of MaMAPK3 decreases the cold tolerance of transgenic plants}

We identified 20 individual equivalent transcripts in Musa genome using the sequences of all Arabidopsis thaliana MAPK genes as a reference (Fig. S1A). The expressions of all MaMAPKs in Musa genome were determined by quantitative real-time PCR (qRT-PCR) after 3 $\mathrm{h}$ of cold stress. Our data indicated that the expression of only MaMAPK3a was dramatically increased in Cavendish banana and 'Dajiao' upon cold stress (Fig. S1B). Furthermore, the expression of MaMAPK $3 a$ in 'Dajiao' was almost five times higher compared with Cavendish banana. As a result, MaMAPK3a (MaMAPK3 as follows) was chosen as the target in this study.

In the present study, we assessed the subcellular localization of MaMAPK3 in banana protoplast according to the fluorescence of green fluorescent protein (GFP). The open reading frame (ORF) of MaMAPK3 was in frame with the GFP N-terminus and C-terminus, and no signal was detected in the MaMAPK3-GFP fusion construct. The green fluorescence was detected in both the nucleus and cytoplasm of the cells transformed with the GFP-MaMAPK3 fusion construct (Fig. S2A), and the same result was found in the cells transformed with the control vector (Fig. S2B). These findings suggested that MaMAPK3 was located in both the nucleus and cytoplasm.

We tried to obtain MaMAPK3 over-expressing line and MaMAPK3-RNAi line of 'Dajiao' plants. However, all MaMAPK3 over-expressing plants showed browning and death during germination as well as plant regeneration of resistant embryos (Fig. S3). Therefore, only transgenic 'Dajiao' plants with reduced expression of MaMAPK3 by RNAi approach could be practically used and reported in the present study. A total of 30 resistant RNAi lines (named from MRi-1 to MRi-30) were obtained. HPT (1025 bp) and sense strand (200 bp) were amplified from 10 (MRi-2, MRi-4, MRi-5, MRi-13, MRi14, MRi-15, MRi-19, MRi-22, MRi-24 and MRi-28) of 30 lines (Fig. S4). Southern blotting analysis showed a single copy in MRi-14, MRi-15 and MRi-22 lines (Fig. S5).

MRi-15 and MRi-22 plants were used for the evaluation of cold resistance. After 5 days of cold stress, 'Dajiao' WT showed a normal phenotype, while the leaves of the two transgenic lines became yellow and showed symptoms of water loss. After 7 days of cold stress, minor injuries were found in 'Dajiao' WT leaves, while the leaves of 'Dajiao' transgenic plants displayed symptoms of severe necrosis and wilting (Fig. 1a). Under most stress conditions, malondialdehyde (MDA) is one of the most representative markers for membrane destruction after free-radical chain reactions [45]. The level of MDA was assessed to investigate the physiological mechanism underlying the decreased cold tolerance of 'Dajiao' transgenic plants. The MDA content of 'Dajiao' WT plants remained stable with the increased duration of cold exposure. In contrast, the MDA content of two 'Dajiao' transgenic lines was significantly increased after cold stress, and such elevation still remained high after recovery for 2 days (Fig. 1b). These results suggested that the suppression of MaMAPK3 negatively regulated the cold resistance of 'Dajiao' plants. The decreased cold resistance in 'Dajiao' transgenic plants indicated that the pathways associated with cold resistance might be negatively regulated. To test this conjecture, the expressions of MaMAPK3 and cold resistance-related genes (including $M Y B 44$, ICE1, MYBS3, DREB1G, DREB1D, COR1 and SPC4) were verified by qRT-PCR under cold stress $\left(10^{\circ} \mathrm{C}\right.$ exposure for $0,3,5$ and 7 days, followed by recovery for 2 days). The expression of MaMAPK3 was markedly increased in 'Dajiao' WT after 3 days of cold stress, while the expression of MaMAPK3 remained unchanged in 'Dajiao' transgenic plants under cold stress (Fig. 2a). Interestingly, the expressions of MYB44, MYBS3, ICE1, COR1 and $S P C 4$ in 'Dajiao' transgenic plants were suppressed to various degrees compared with the 'Dajiao' WT plants (Fig. 2b-f). The expressions of DREB1G and $D R E B 1 D$ in 'Dajiao' transgenic plants were not affected under the normal conditions. However, the expression of DREB1G in 'Dajiao' WT plants was dramatically increased compared with the 'Dajiao' transgenic plants under cold stress (Fig. $2 \mathrm{~g}$ and $\mathrm{h}$ ). 


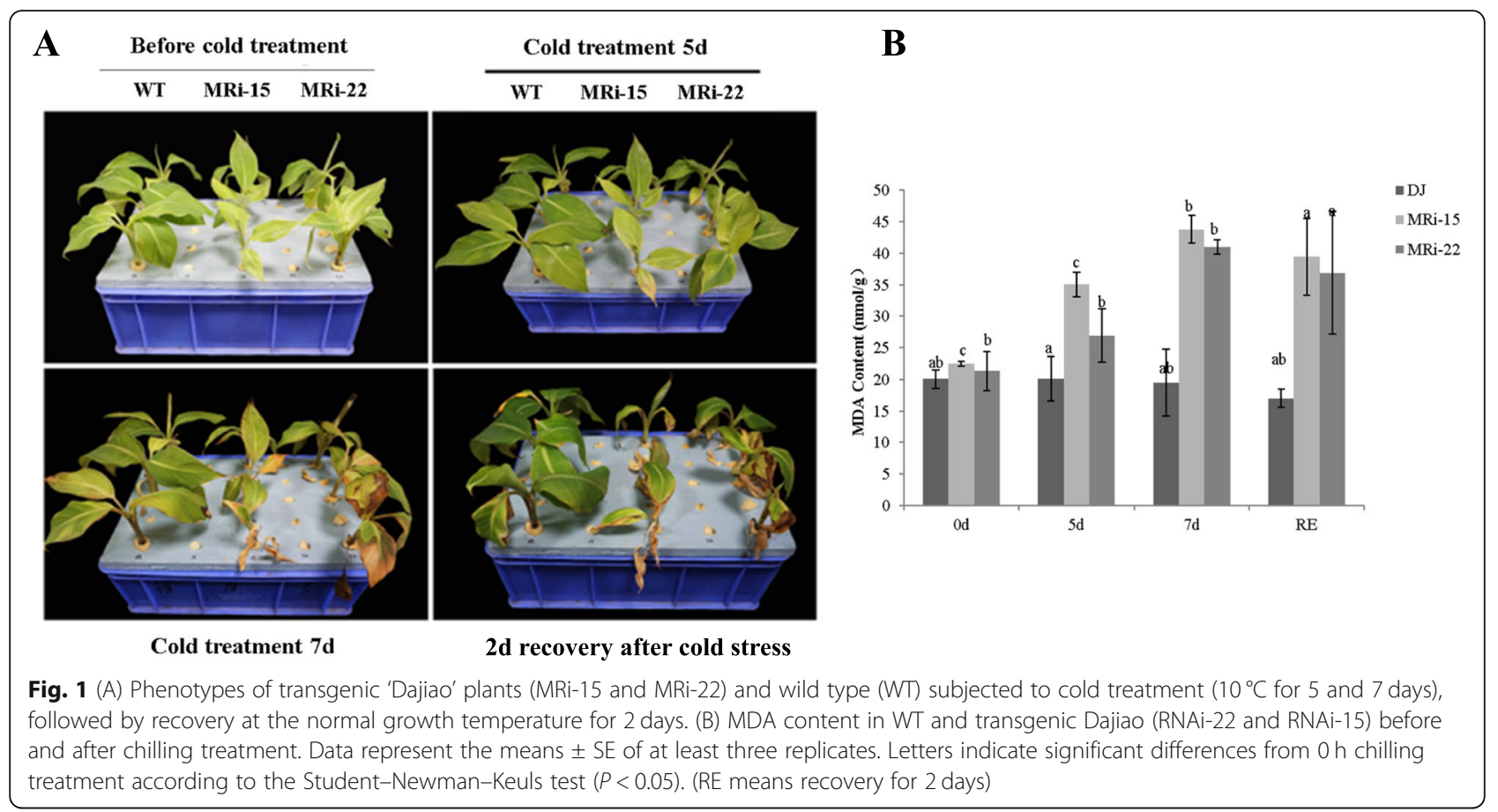

\section{MaMAPK3 interacts with MaICE1}

Since the expression of MaICE1 is suppressed in MaMAPK3 RNAi 'Dajiao' transgenic plants and the functions of ICE1 are affected by MAPK3 in Arabidopsis thaliana and rice [21, 23], we carried out a yeast twohybrid $(\mathrm{Y} 2 \mathrm{H})$ assay to explore the potential relationship between MaICE1 and MaMAPK3. Strong self-activation activity was detected from the full-length MaICE1 protein when binding to the pGBKT7 vector, whereas ADMaICE1 (MaICE1 cloned into pGADT7) showed no self-activation activity. Therefore, we chose MaICE1 and cloned it into the pGADT7 vector in our assay. The result of the $\mathrm{Y} 2 \mathrm{H}$ assay demonstrated the interaction of MaMAPK3 and MaICE1 (Fig. 3a). We performed bimolecular fluorescence complementation (BiFC) assays to further explore the interplay between MaICE1 and MaMAPK3 in vivo. The results indicated that the fluorescence signal was not detected in CEYFP-MaICE1/ nEYFP and nEYFP-MaMAPK3/cEYFP (the negative combinations), while co-expression of cEYFP-MaICE1 and nEYFP-MaMAPK3 yielded strong signals in the nucleus (Fig. 3b). Such fluorescence detection indicated a direct protein-protein interaction between MaICE1 and MaMAPK3, which further supported our Y2H results. The function of MaICE1 was further investigated and discussed in subsequent experiments.

Overexpression of MalCE1 increases the cold tolerance of transgenic plants

The full-length cDNA encoding MaICE1 (GenBank accession no. KM379133) was isolated from 'Dajiao'. The subcellular localization of MaICE1 was investigated in banana protoplast according to GFP fluorescence. The ORF of MaICE1 was in frame with the GFP N-terminus and $\mathrm{C}$-terminus, and no signal was detected in the MaICE1-GFP fusion protein. The GFP-MaICE1 fusion protein was located in the nucleus, whereas the GFP protein in the control was detected in both the cytoplasm and nucleus (Fig. S6).

A total of 13 resistant MaICE1-overexpressing lines were obtained. HPT (1025 bp) and ICE1 (200 bp) were amplified from 11 of 13 lines (Fig. S7A and B). Two individual over-expression Cavendish banana lines (\#11 and \#13) showing a single copy (Fig. S7C) and a high level of MaICE1 expression (Fig. S7D) were used for cold treatment analysis, along with Cavendish banana WT and 'Dajiao' WT plants. Cavendish banana WT, 'Dajiao' WT and 2-month-old MaICE1-overexpressing transgenic lines (\#11 and \#13) were exposed to a temperature of $10^{\circ} \mathrm{C}$ for $48 \mathrm{~h}$, followed by recovery at normal temperatures. Under normal conditions, the phenotype of transgenic plants could be distinguished between the 'Dajiao' WT and Cavendish banana WT plants. When the plants were exposed to $10^{\circ} \mathrm{C}$ for $24 \mathrm{~h}$, the leaves of Cavendish banana WT plants showed severe cold injury, whereas only minor injury was observed in the transgenic and 'Dajiao' WT plants (Fig. 4a). After 3 days of recovery under the normal condition, the cold injury area of \#11 (35.7\%), \#13 (38.9\%) and 'Dajiao' WT (32.6\%) plants was dramatically improved compared with the WT plants (93.2\%) (Fig. 4b). 

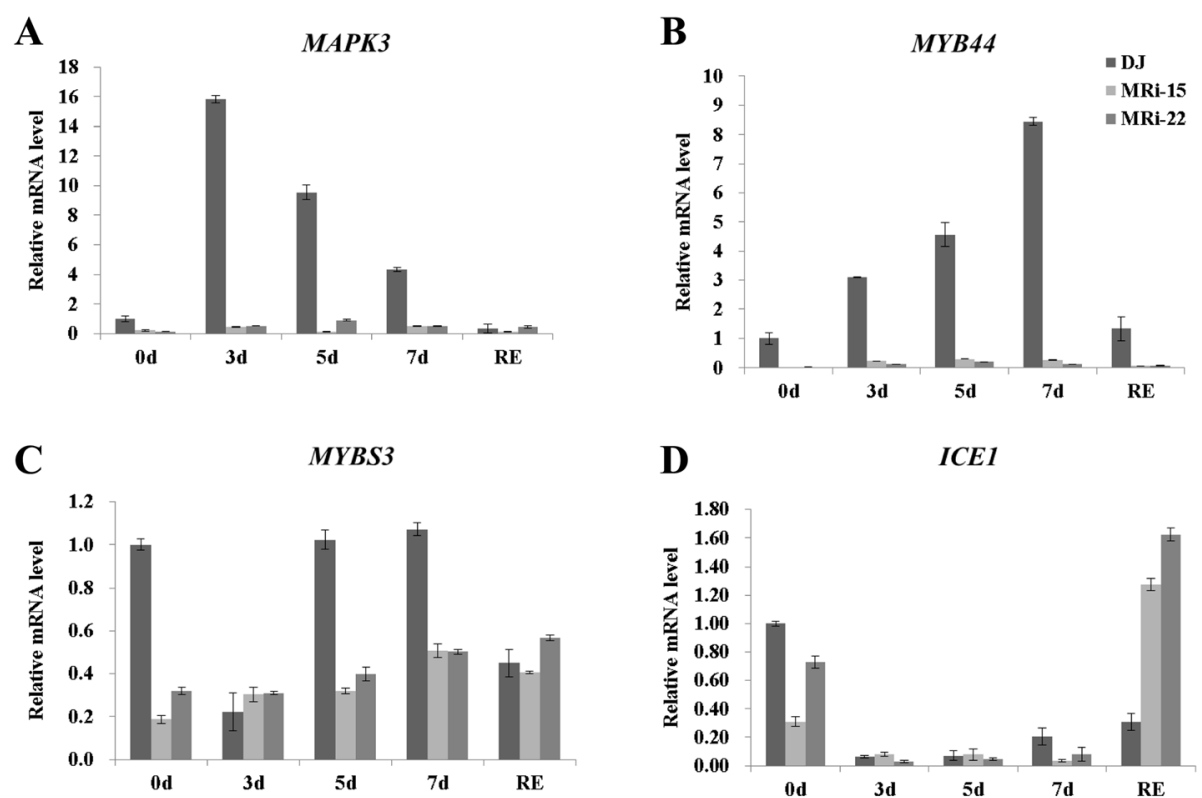

$\mathrm{D} \quad$ ICE1
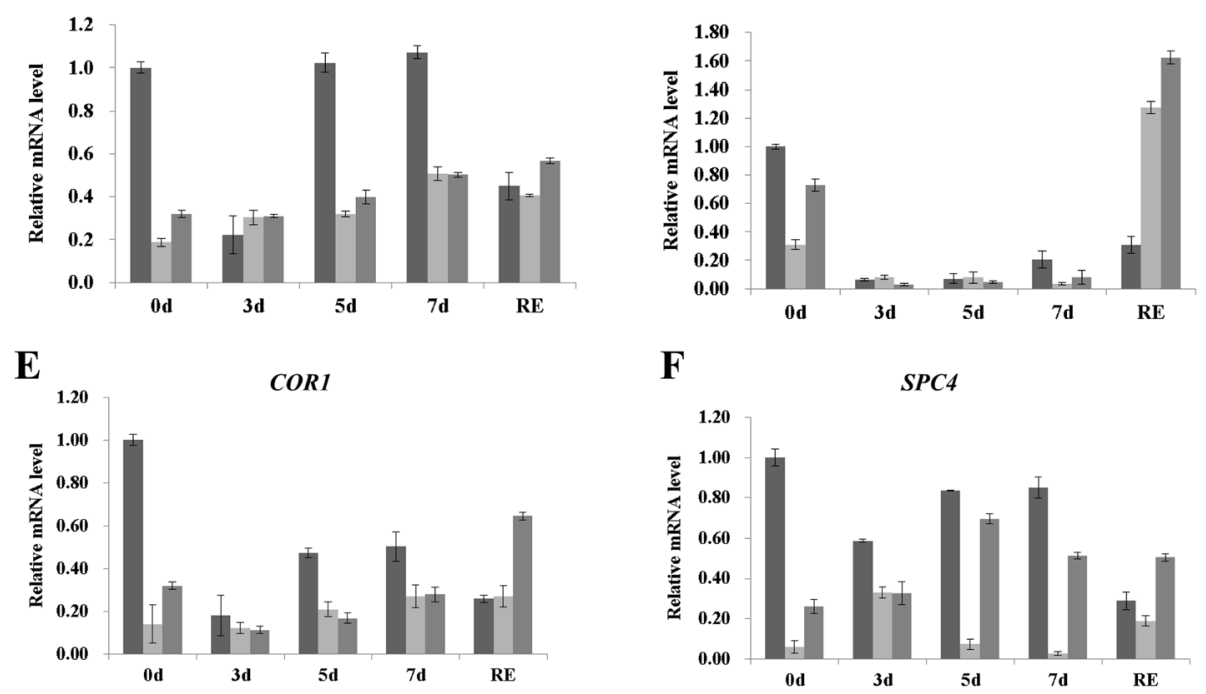

$\mathbf{F}$
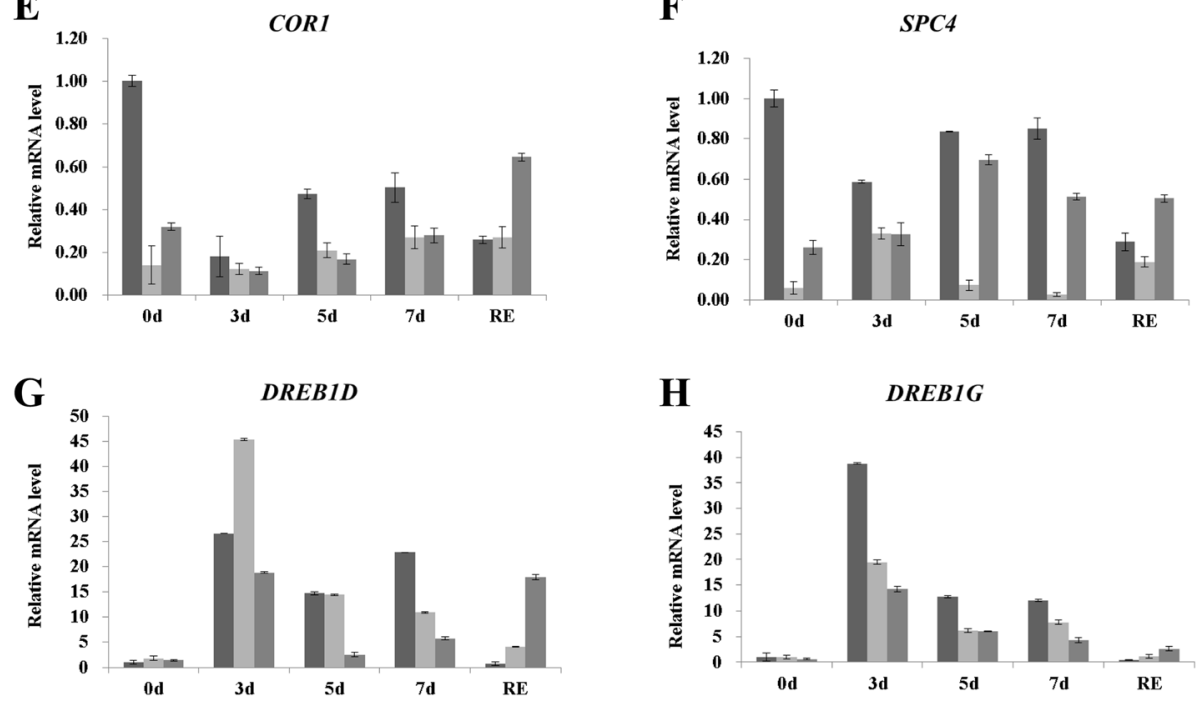

Fig. 2 Analysis of relative expression levels of MAPK3 and cold-relative genes by quantitative real-time PCR in wild type and transgenic 'Dajiao' lines (MRi-15 and MRi-22) before and after cold treatment. Data represent the means \pm SE of at least three replicates. Letters indicate significant differences from $0 \mathrm{~h}$ cold treatment according to the Student-Newman-Keuls test $(P<0.05)$ (RE means recovery for 2 days)

MDA level, proline (Pro) content, and electrolyte leakage were analyzed to investigate the physiological and biochemical mechanisms underlying the improved cold tolerance of MaICE1 over-expressing banana plants. Figure $4 \mathrm{c}$ shows that after the cold stress, the electrolyte leakage of 'Dajiao' WT and transgenic plants was markedly decreased compared with the Cavendish banana WT plants. After exposure to $10^{\circ} \mathrm{C}$ for 2 days, all examined plants showed increased levels of Pro. Nevertheless, the content of Pro in transgenic plants was dramatically greater compared with the Cavendish banana WT plants (Fig. 4d).
Figure 4e shows that the accumulation of MDA was remarkably lower in the MaICE1 transgenic lines and 'Dajiao' WT plants compared with the Cavendish banana WT plants at the end of the cold stress. These findings supported that the over-expression of MaICE1 could improve the cold resistance in Cavendish banana.

As a TF, MaICE1 may regulate a variety of downstream elements that can noticeably improve cold resistance in the Cavendish banana over-expression lines. To verify such a hypothesis, we performed a transcriptomic assay to compare the expression profiles of WT and one 
A

AD

AD-ICE1

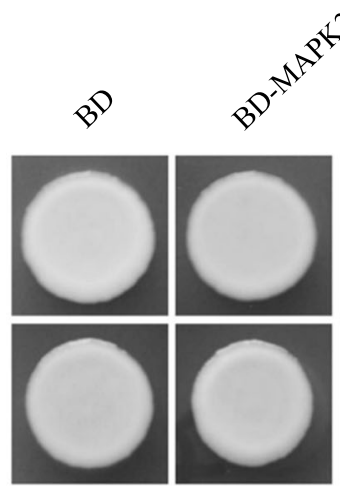

SD-Leu-Trp
8
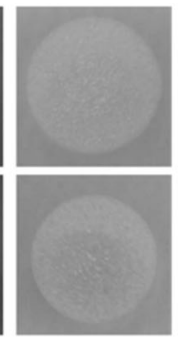

SD-Leu-Trp-His-Ade

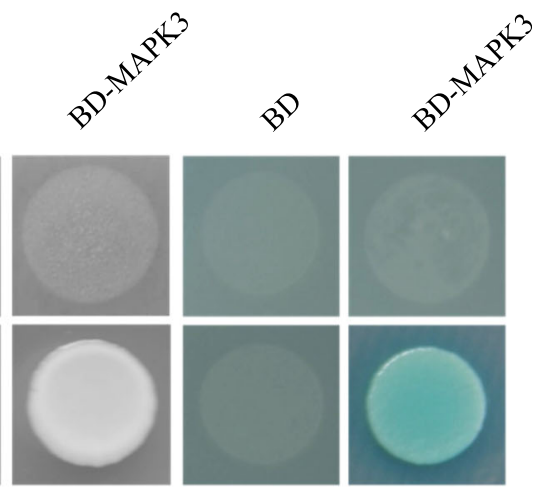

X-gal-assay

B

$\mathbf{a}$

WT1: pSTA1-cEYFP-ICE1 + pSTA4-nEYFP

WT2: pSTA4-nEYFP-MAPK3a + pSTA1-cEYFP

$$
\text { MAPK3a+ICE1: pSTA1-cEYFP-ICE1 + pSTA4-nEYFP-MAPK3a }
$$

b
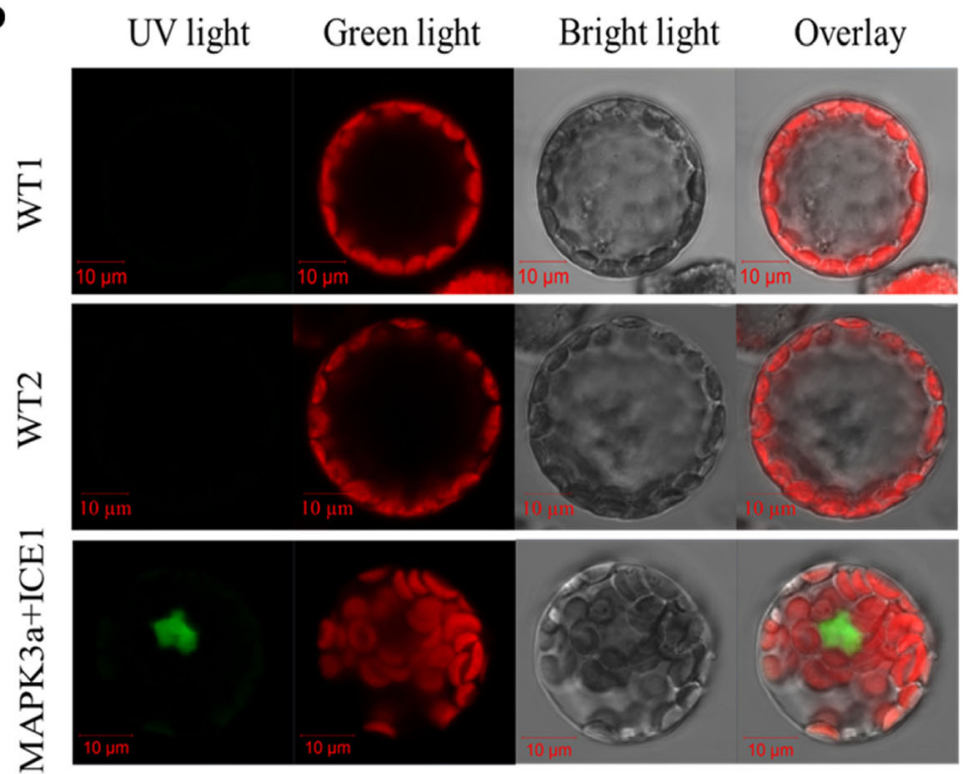

Fig. 3 (A) Physical interactions between MalCE1 and MaMAPK3 using Y2H Assays. (The sequences of MalCE1 and MaMAPK3 were cloned from 'Dajiao') (B) Biomolecular fluorescence complementation visualization of the interaction between MaMAPK3 and MalCE1. (B-a) Schematic diagrams of the constructs used for biomolecular fluorescence complementation assay. The WT1 and WT2 were two negative controls, respectively. (B-b) The images indicate an interaction between MaMAPK3 and MaICE1 by biomolecular fluorescence complementation

over-expression line (\#13) before and after cold stress. A total of 222 and 496 genes were up-regulated and downregulated in \#13 compared with the Cavendish banana WT plants without cold treatment, respectively, when a 2-fold change was used as a cutoff threshold (Table S1). However, 526 and 196 genes were up-regulated and down-regulated, respectively, in line \#13 compared with the Cavendish banana WT plants after only $1 \mathrm{~h}$ of cold exposure (Table S2). After $4 \mathrm{~h}$ of cold stress, 1109 upregulated genes and 540 down-regulated genes were identified in line \#13 compared with the Cavendish banana WT plants (Table S3), indicating that the gene expression profile was profoundly altered in the transgenic plants. In these differentially expressed genes (DEGs), many genes involved in cold response showed significant up-regulation in transgenic banana under cold stress, 
A

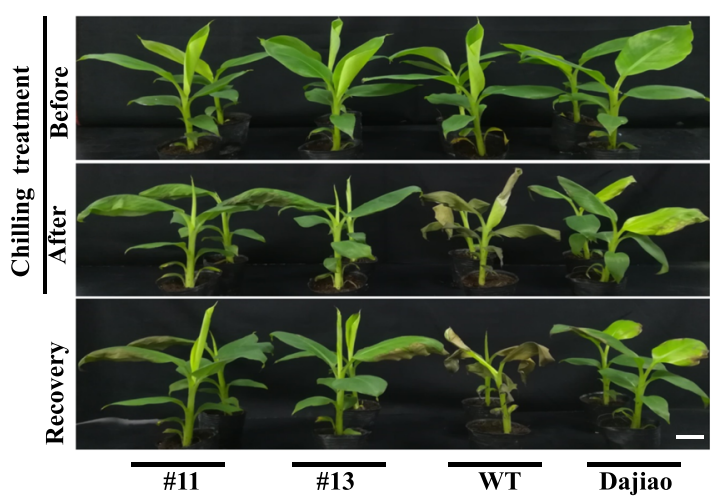

B

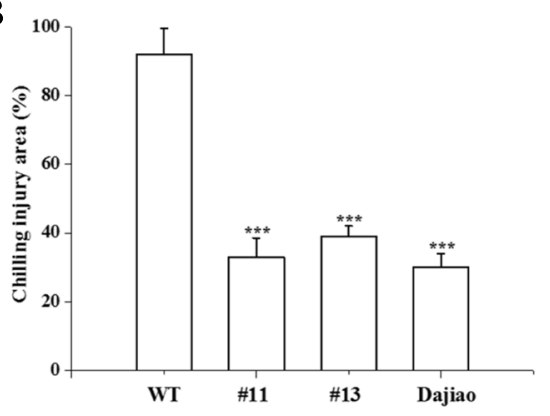

D

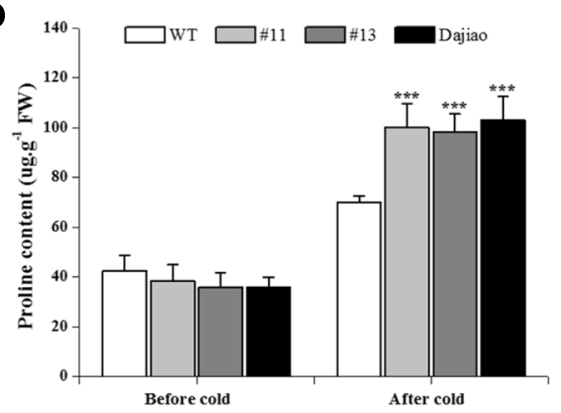

C

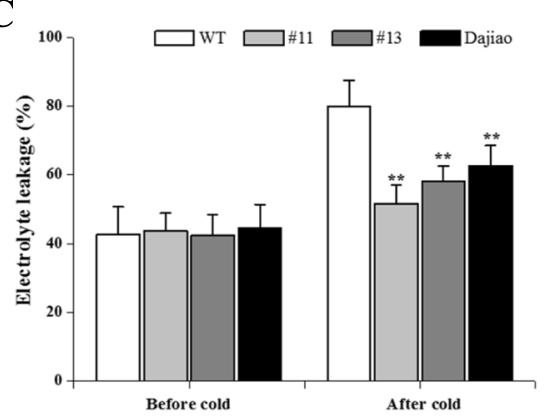

$\mathbf{E}$

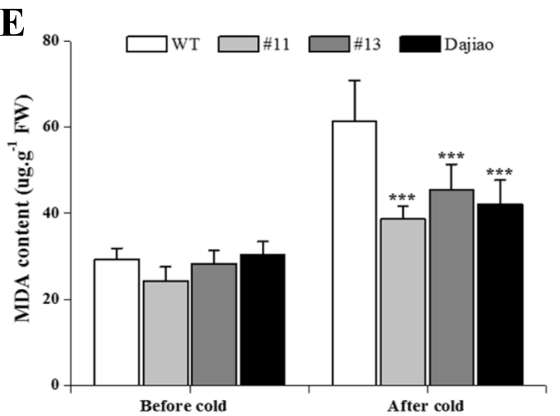

Fig. 4 Cold tolerance assay of transgenic Cavendish banana plants. (A) Phenotypes of transgenic Cavendish banana plants (\#11 and \#13), 'Dajiao' and the wild-type (WT) before and after cold treatment $\left(10^{\circ} \mathrm{C}\right.$ for $48 \mathrm{~h}$ ), followed by recovery in an ambient environment for 3 days (B) Injured area of WT banana and transgenic plants analyzed after cold treatment (bar: $3.5 \mathrm{~cm}$ ). Effect of MalCE1 expression in banana on levels of (C) electrolyte leakage, (D) pro and (E) MDA. Two-month-old banana plants of WT, 'Dajiao' and transgenic Cavendish banana plants (\#11 and \#13) were exposed to $10^{\circ} \mathrm{C}$ for $48 \mathrm{~h}$. Leaves were collected before and after cold treatment. FW, fresh weight. Each value represents the means of three biological replicates, and vertical bars indicate the SE. ${ }^{* *} P<0.01$. ${ }^{* *} P<0.001$

such as SPC4 (Ma03_g02760) and COR2 (Ma06_ g36560). To find out the effect of MaICE1 overexpression on MAPK cascades, the expressions of all identified MAPK cascade genes in the transcriptome were analyzed. The expressions of MaMAPKK2 and MaMAPK3 were significantly changed in transgenic plants compared with the WT plants under cold stress (Table S4). In Cavendish banana WT plants, the expression of MaMAPKK2 was down-regulated under cold stress, while its expression in transgenic plants was significantly up-regulated under cold stress. The expression of MaMAPK3 was markedly increased in Cavendish banana WT and transgenic plants after $1 \mathrm{~h}$ of cold stress.
After $4 \mathrm{~h}$ of cold stress, the expression of MaMAPK3 was decreased in Cavendish banana WT plants, while it remained at a high level in transgenic plants (Table S4).

Additionally, out of these DGEs, a Peroxidase P7 (POD P7) (ID NO. Ma10_g27800) drew our particular attention. Under the normal condition, the expression of MaPOD P7 was significantly up-regulated in line $\# 13$ compared with Cavendish banana WT plants, while its expression remained at a high expression level during cold treatment. In our previous research, this MaPOD P7 protein is localized in the plasma membrane and chloroplast, which plays a critical role in the cold resistance of 'Dajiao' (He et al., 2018). Besides, the interaction 
between MaICE1 and MaPOD P7 was confirmed by $\mathrm{Y} 2 \mathrm{H}$ systems (Fig. 5a) as well as BiFC assays (Fig. 5b).

\section{Antioxidant capacity analysis in the transgenic plants} POD P7 is a gene related to antioxidant capacity. To evaluate antioxidant capacity in Cavendish banana WT and MaICE1-overexpressing transgenic plants, $\mathrm{H}_{2} \mathrm{O}_{2}$ and $\mathrm{O}_{2}{ }^{-}$contents in Cavendish banana WT and MaICE1-overexpressing transgenic Cavendish banana leaves were determined by 3,3'-diaminobenzidine (DAB) and Nitroblue Tetrazolium (NBT) staining. Our data indicated that the $\mathrm{H}_{2} \mathrm{O}_{2}$ and $\mathrm{O}_{2}^{-}$contents were significantly lower in transgenic Cavendish banana plants compared with Cavendish banana WT plants under cold stress (Fig. 6). According to these findings, we speculated that MaICE1-MaPOD P7 interaction played a fundamental role in cold resistance in MaICE1-overexpressing transgenic Cavendish banana.

Since POD P7 was found to be significantly changed in transgenic Cavendish banana with MaICE1 overexpression and there was an interaction between MaMAPK3 and MaICE1, we hypothesized that a decreased oxidoreductase activity occurred in MaMAPK3
RNAi transgenic 'Dajiao' plants with the inhibition of MaMAPK3 by RNAi. The POD activity of 'Dajiao' WT and transgenic 'Dajiao' plants was examined. Under the normal condition, the POD activity was higher in transgenic 'Dajiao' plants compared with 'Dajiao' WT plants (Fig. 7a). However, after cold treatment, the POD activity remained stable in 'Dajiao' WT plants, while it was significantly decreased in transgenic 'Dajiao' plants (Fig. 7b). To figure out the reason why the POD activity was higher in MaMAPK3 RNAi transgenic 'Dajiao' plants, we measured the $\mathrm{H}_{2} \mathrm{O}_{2}$ and $\mathrm{O}_{2}{ }^{-}$contents by DAB and NBT staining. We found that the contents of $\mathrm{H}_{2} \mathrm{O}_{2}$ and $\mathrm{O}_{2}{ }^{-}$were dramatically greater in transgenic 'Dajiao' plants compared with the 'Dajiao' WT plants under the normal condition (Fig. 7c and d), which might explain the greater POD activity in transgenic 'Dajiao' plants under the normal condition.

\section{Discussion}

Plants have evolved a variety of sophisticated defense mechanisms to deal with different stresses. In plants, complex and rapid signaling pathways are responsible for the response and adaptation to cold stress, and such pathways can impair a wide range of cellular activities, such as

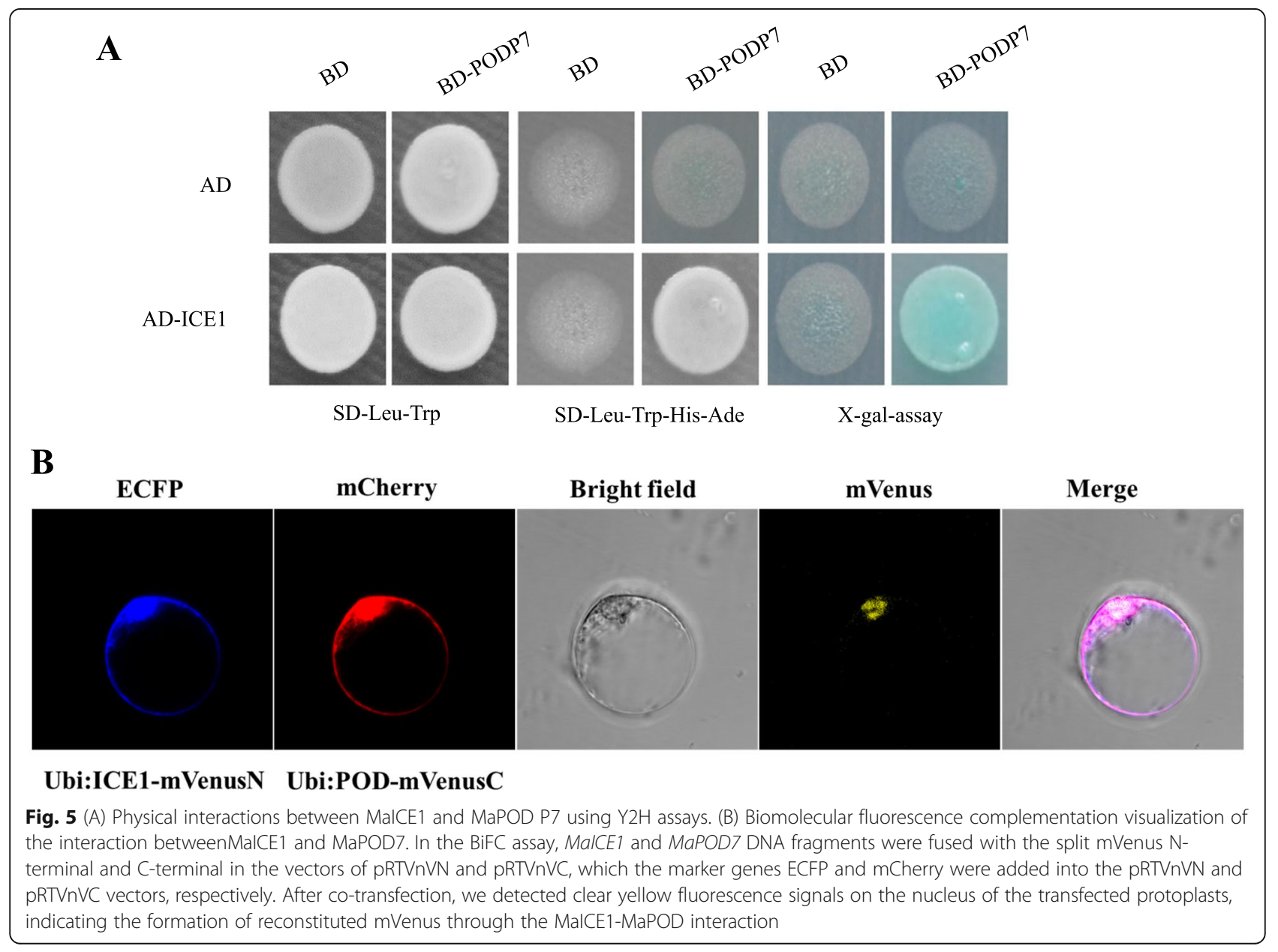




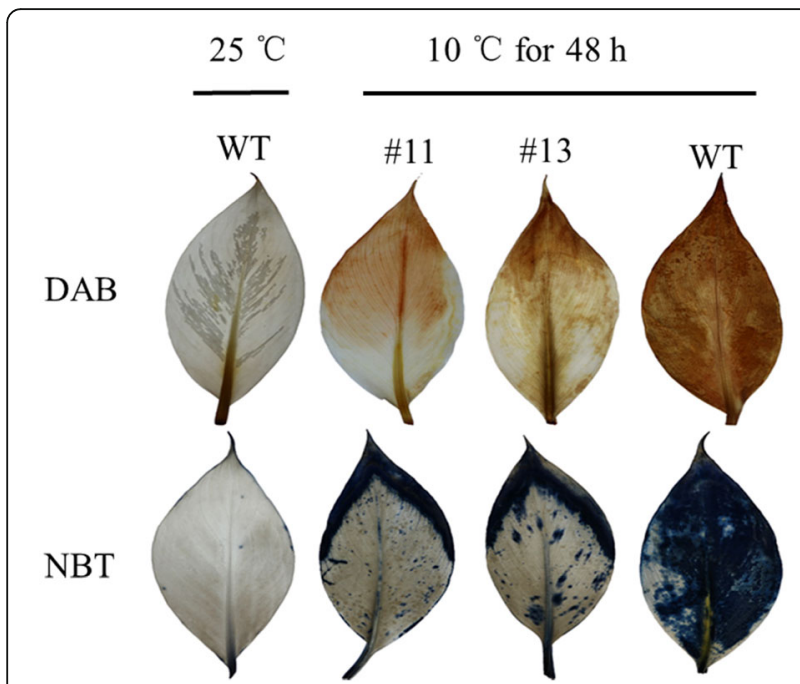

Fig. 6 Nitroblue tetrazolium (NBT), and 3,3'-diaminobenzidine (DAB) to analyze the accumulation of $\mathrm{O}_{2}{ }^{-}$and $\mathrm{H}^{2} \mathrm{O}^{2}$ in the transgenic Cavendish banana (\#11and \#13) and wild type plants

physiology or biochemistry changes, transcriptional regulation, cell division and morphogenesis, protein folding and metabolic changes in nutrient flux [46-48]. In our lab, we have investigated the molecular mechanisms of cold resistance in bananas for a long time. Based on the previous results from proteomics, transcriptomics, gene function analysis, membrane proteomics, and phosphoproteomics $[40-43,49]$, we have found several independent pieces of evidence for mechanisms underlying the cold resistance. In the present study, we aimed to investigate the potential connections for those independent mechanisms.

\section{MaMAPK3 is a positive regulator of cold tolerance}

Transcriptome analysis has indicated that the expression of MaMAPK3 in 'Dajiao' is significantly higher compared with Cavendish banana under cold stress [42]. The pathways mediated by MAPKs not only display an important effect on the development of plants but also participate in regulatory responses to stresses [10,50,51]. MAPK family is divided into four subgroups based on sequence and structural similarity, namely $\mathrm{A}, \mathrm{B}, \mathrm{C}$, and D subgroups [52]. It is well known that MAPKs belonging to A and B subgroups participate in the response to biological and abiotic stresses. In rice, OsMAPK3 belonging to subgroup A acts as a positive regulator by interacting with the phosphorylated OsbHLH002/ICE1 protein [21], while AtMAPK3 has been reported as a negative regulator in cold resistance of Arabidopsis thaliana [20, 23]. In our present study, MaMAPK3 was activated by cold stress, and the phylogenetic tree analysis revealed that MaMAPK3 belonged to subgroup A (Fig. S1). Therefore, we hypothesized that MaMAPK3 participated in the regulation of cold resistance in 'Dajiao' that has a stronger cold tolerance than other banana cultivars.

We investigated MaMAPK3 using both knock-in and knock-down (by RNAi) transgenic lines of MaMAPKs. We did not successfully establish the over-expression lines as apparently too much MaMAPK3 protein was likely to disturb the balance of growth. However, the transgenic plants with suppressed expression of MaMAPK3 showed dwarf features and cold sensitivity compared with the WT plants. Moreover, the phenotype analysis of transgenic plants allowed us to conclude that MaMAPK3 indeed participated in cold response and positively regulated cold resistance in "Dajiao". To understand the effect of MaMAPK3 RNAi on the global transcriptional level, we first investigated the expression profiles of seven marker genes [41, 48, 53-56], which have been well known to participate in cold resistance, in the transgenic lines. Our data indicated that the suppression of MaMAPK3 exerted a negative effect on cold resistance of "Dajiao" at the transcriptional level. Besides, we found that the reduced oxidoreductase activity in transgenic 'Dajiao' could further rationalize and connect to the importance of POD function. It should be noted that MAPK3 might play different roles in different species. In Arabidopsis thaliana, only AtMAPK4/6 and AtMAPK3 can interact with AtICE1, and AtMAPK3 functions as a negative modulator under cold stress [20, 23], whereas 10 of 22 MAPKs in banana can interact with MaICE1 (Fig. S8). These results indicated that there was a large difference between Arabidopsis thaliana and banana in terms of MAPK3-mediated regulatory mechanism.

\section{MaICE1 is a positive regulator of cold tolerance}

The largest TF family in plants is composed of bHLH proteins [54, 57]. A great deal of evidence has shown that bHLH TFs have fundamental functions in plant responses to different abiotic stresses, such as drought stress [58], salinity stress [59], and cold stress [35, 37, 54, 60]. The bHLH proteins consist of conserved bHLH signature domains composed of one basic region at the $\mathrm{N}$-terminal end and one HLH region at the C-terminal end [24, 38]. The specificity of the DNA-protein interactions is determined by the basic region consisting of approximately 15 amino acids, including several basic residues [37]. There are two amphipathic $\alpha$-helices in the HLH region, which are connected by a loop region of variable length and play a critical role in the formation of homodimers or heterodimers [24]. The basic region allows bHLH TFs to bind to consensus E-box (5'-CANNTG-3') or G-box (5'-CACG TG-3') cis elements to regulate the gene expression [25]. As TFs, MYC-type proteins are localized in the nucleus to exert their regulatory functions, and most isolated bHLH proteins are localized in the nucleus [37, 54, 61]. Similarly, 


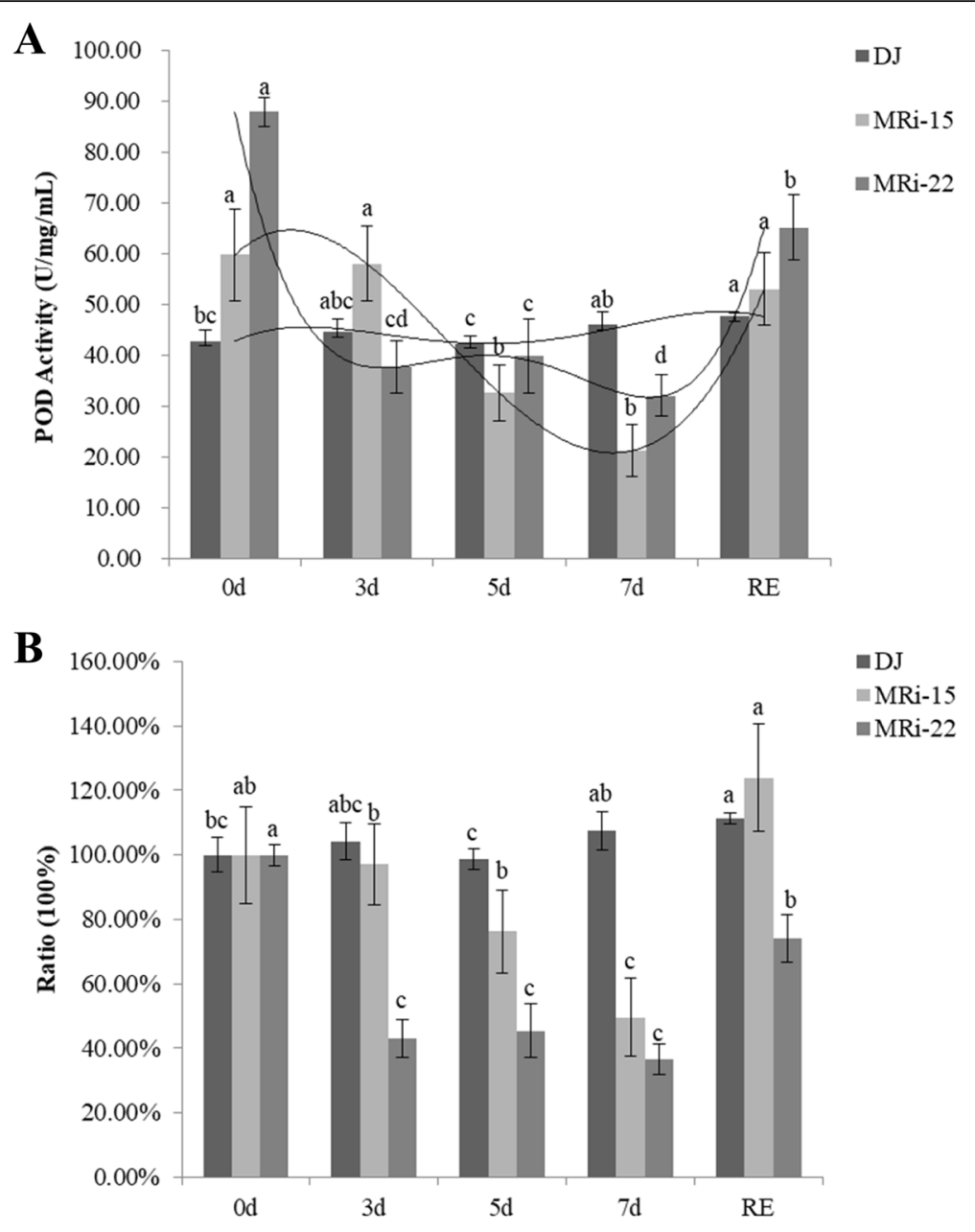

C

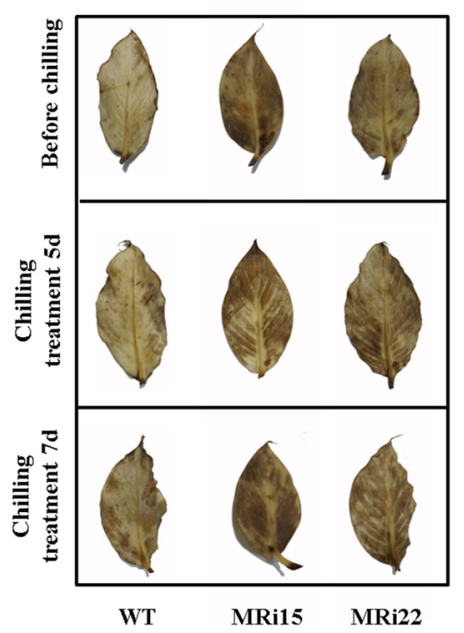

D

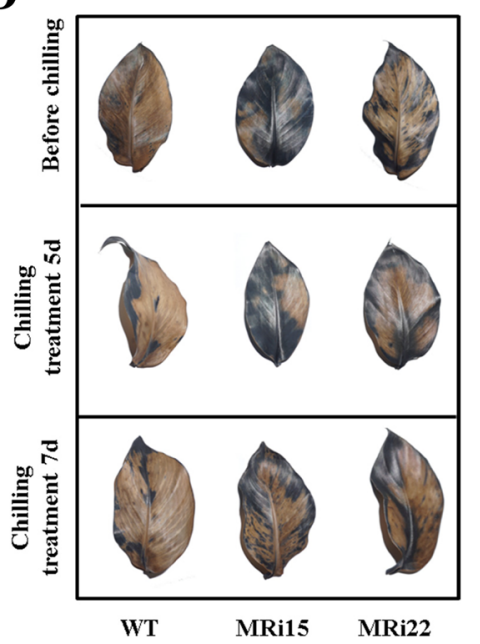

Fig. 7 (A) POD activity, (B) the ratio of POD activity, (C) DAB straining, (D) NBT straining to analyze the accumulation of $\mathrm{O}_{2}^{-}$and $\mathrm{H}^{2} \mathrm{O}^{2}$ in wild type and transgenic 'Dajiao' (RNAi-22 and RNAi-15) before and after cold treatment. Data represent the means \pm SE of at least three replicates. Letters indicate significant differences from $0 \mathrm{~h}$ cold treatment according to the Student-Newman-Keuls test $(\mathrm{P}<0.05)$ (RE means recovery for 2 days) 
MaICE1 was confirmed to be localized in the nucleus after the transformation of banana protoplasts (Fig. S6), suggesting that MaICE1 was a nuclear protein.

For now, the roles of bHLH homologs in 'Dajiao', a very cold-hardy plant, remain largely unexplored. Compared with the Cavendish banana, 'Dajiao' is significantly cold-tolerant in winter, which can undergo low temperatures down to $0-4{ }^{\circ} \mathrm{C}[41,49]$. Therefore, it is highly necessary to characterize the functions of bHLH genes from 'Dajiao' to clarify the cold signaling pathway associated with cold resistance and identify useful gene candidates for genetic manipulation [41]. We transformed MaICE1 into a cold-sensitive perennial plant, Cavendish banana with great agronomic value. Constitutive expression of MaICE1 led to significant changes in the cold resistance of the transgenic plants. Over-expression of MaICE1 in banana significantly decreased the cold stressinduced damage (Fig. 4a) and resulted in better plant phenotypes compared with the WT plants after cold stress (Fig. 4b). These above-mentioned findings were also accompanied by an elevated level of Pro (Fig. 4d), reduced MDA content (Fig. 4e) and electrolyte leakage (Fig. 4c). Collectively, our results showed that MaICE1 played a beneficial role in cold resistance. Previous studies have shown that banana plants over-expressing stressresponsive TFs, such as AtCBF1 and MusabZIP5, frequently exhibit growth restriction $[41,44]$. However, except that the growth period was 1 month longer than WT plants, no apparent phenotypic changes were caused by MaICE1 over-expression compared with the WT plants under normal growth conditions, implying that MaICE1 could be potentially used in genetic manipulation to ameliorate cold resistance in banana.

It is well known that as a highly complex process, the stress response is mediated by several signaling pathways [41]. To clarify the molecular mechanisms underlying the improved cold resistance, we compared transcriptional profiles between Cavendish banana WT plants and the over-expression transgenic line (\#13) under normal conditions and cold stress. We found that comprehensive transcriptomic modifications were induced in the transgenic line by the over-expression of MaICE1. It is worth noting that MaICE1 both promotes and inhibits the expressions of many genes at the mRNA level, suggesting both positive and negative effects on the expression atlas. Such a finding is not an exception since many studies have reported the extensive transcriptional reprogramming in transgenic plants over-expressing a TF compared with their WT controls $[37,62]$. In all DEGs found in transcriptomics, the expression of POD P7 (Ma10_g27800) exhibited the largest difference before and after the cold stress. In our previous quantitative proteomics and membrane proteomics analyses between cold-tolerant 'Dajiao' and cold-sensitive Cavendish banana, POD P7 protein is the only peroxidase with an increased abundance localized in the plasma membrane and chloroplast. The increased POD P7 expression appears to be a key cellular adaptation contributing to the cold tolerance of 'Dajiao' by involving in decreased lipid peroxidation [49]. Besides, in our new proteomics data (data not shown), the abundances of four SOD and 27 POD proteins were significantly increased in MaICE1overexpressing Cavendish banana plants, and 10 of 27 PODs were POD-P7 (including Ma10_g27800) or PODP7 like proteins. Moreover, the interaction between MaICE1 and MaPOD P7 was confirmed by $\mathrm{Y} 2 \mathrm{H}$ systems and BIFC assays (Fig. 5), indicating that ICE1-POD P7 interactions played a key role in cold tolerance in transgenic banana.

As shown in Supplementary Table 1, 2 and 3, the number of DEGs after $4 \mathrm{~h}$ of cold stress (1109 upregulated and 540 down-regulated genes) was significantly higher compared with the normal conditions (222 and 496, respectively). We speculated that MaICE1 underwent certain undetermined modifications upon exposure to cold stress, leading to the expression or suppression of a set of stress-responsive genes involved in the plant stress-responsive signaling network. For now, it has been reported that the activity of ICE1 can be positively regulated by the phosphorylation of OST1 [63] and negatively mediated by the phosphorylation of MAPK3 [20, 23] in Arabidopsis thaliana under cold stress. Based on the $\mathrm{Y} 2 \mathrm{H}$ and BiFC assays, MaMAPK3 was found to interact with MaICE1 (Fig. 3). The results indicated that MaMAPK3-MaICE1 played a central role in cold response in banana.

\section{MaMKK2 interacts with MaMAPK3}

MKK2 is an intermediate node in MAРККK-MAРКKMAPK cascades. Over-expression of MKK2 affects the expression of the CBF gene by phosphorylation of downstream MPK6/MPK4 to enhance the cold tolerance of Arabidopsis [15]. In banana, however, we found that the phosphorylation level of MaMKK2 was significantly increased in cold-resistant 'Dajiao', while such elevation was not detected in cold-sensitive banana. These results revealed the important role of MaMKK2 in cold resistance of banana [40]. After we found that MAPK3 RNAi 'Dajiao' plants showed a phenotype with decreased cold resistance, the $\mathrm{Y} 2 \mathrm{H}$ method was used to assess the interplay between MKK2 and MAPK3, and we found that MaMKK2a could indeed interact with MaMAPK3 (Fig. S11). Therefore, based on the significantly up-regulated expressions of MKK2 and MAPK3 genes in ICE1 over-expressing transgenic plants, we believed that the interaction between MKK2 and MAPK3 was most likely involved in the cold resistance of banana. 


\section{Conclusions}

Collectively, the MAPK3-ICE1-POD pathway played a critical role in banana cold resistance. Based on the findings acquired from this study, we proposed a model towards a cold resistance mechanism by the MAPK3ICE1-POD pathway (Fig. 8). Under cold stress, MEKK?MKK2-MPK3 cascades were rapidly activated. Subsequently, the ICE1 expression was promoted by the activated MAPK3, thus facilitating the expression of membrane-associated gene POD P7 gene. The MKK2MPK3-ICE1-POD P7 pathway positively affected cold response.

\section{Methods}

Plant materials, growth conditions, and cold treatments 'Dajiao' (Musa spp. "Dajiao"; ABB group; accession No. LCDJ_01) and Cavendish banana (Musa spp. AAA group) were harvested from the National Banana Germplasm Repository, Institute of Fruit Tree Research, Guangdong Academy of Agricultural Sciences. For cold treatment, the potted plantlets were cultivated up to the five-leaf-stage in a growth chamber under the conditions as follows: temperatures of $30 / 28^{\circ} \mathrm{C}$ (day/night), the photon flux density of $240 \mu \mathrm{mol} \mathrm{m} \mathrm{m}^{-2} \mathrm{~s}^{-1}, 12-\mathrm{h}$ photoperiod, and relative humidity of $60-80 \%$. Three plants with a uniform growth status were then placed into a chamber set at $10^{\circ} \mathrm{C}$ for $0,1,3,6,24$ and $48 \mathrm{~h}$ in the dark. The temperature treatment repeated three times in a single growth chamber. The fully expanded leaves on the top of the plants/plantlets were harvested at the indicated time intervals, frozen in liquid nitrogen right away, and preserved at $-80^{\circ} \mathrm{C}$ before further analysis. All specimens at each time point were collected in triplicate.
Three plantlets constitute a biological for each separate cultivar used in this study.

\section{Gene isolation and sequence analysis}

Total RNA was extracted from 'Dajiao' leaves by a plant RNA extraction kit (Code No. 9767, TaKaRa, Dalian, China), and then $1 \mu \mathrm{g}$ purified RNA was reversely transcribed into cDNA using a PrimeScript RT Reagent Kit according to the manufacturer's protocol (TaKaRa). According to the cDNA sequence of MaICE1 obtained from the 'Dajiao' transcriptome data [43], partial 'Dajiao' ICE1 cDNA fragments were amplified by PCR using primers harboring SpeI and BamH I restriction sites (GSP1, Table S5). The sequence of MaMAPK3 was acquired from the genome database of Musa (http:// banana-genome-hub.southgreen.fr/home) [1]. Sequences were aligned using ClustalW [64], and the phylogenetic tree was constructed using MEGA 7 software [65].

\section{Sub-cellular localization}

The ORF of MaMAPK3 was in frame with the GFP Nterminus and $\mathrm{C}$-terminus, and no signal was detected in the MaMAPK3-GFP fusion construct, the signal of GFPMaMAPK3 was used. The full-length cDNA of MaMAPK3 was subcloned into the pMD18-T vector (TaKaRa). Plasmid pMD18-T containing MaMAPK3 was amplified using primers GSP3 (Table S5) containing $\mathrm{Sal}$ I and ClaI restriction sites to assess the subcellular localization of MaMAPK3. The ORF of MaICE1 was in frame with the GFP N-terminus and $\mathrm{C}$-terminus, and no signal was detected in the MaICE1-GFP fusion construct, the signal of GFP-MaICE1 was used. The fulllength cDNA of MaICE1 was subcloned into the

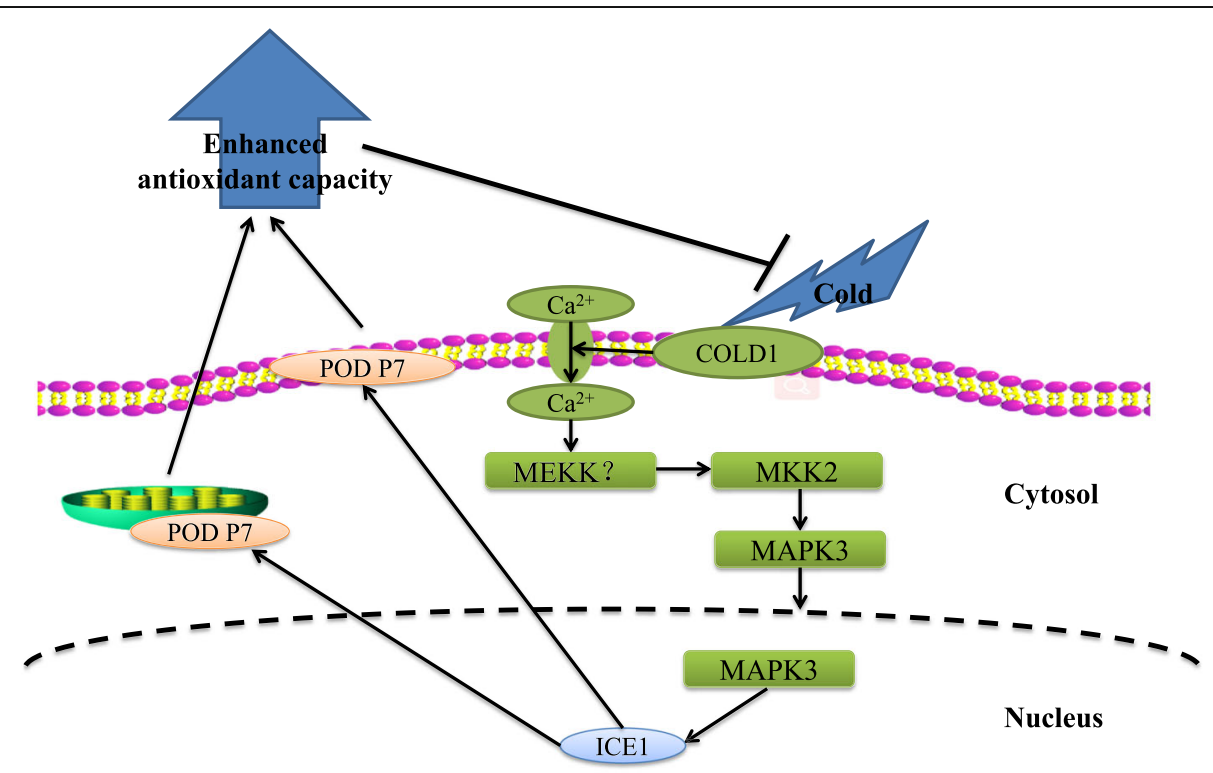

Fig. 8 A working model showing the roles of MAPK3-ICE1-POD P7 pathway in 'Dajiao' cold stress response 
pMD18-T vector (TaKaRa). Plasmid pMD18-T containing MaICE1 was amplified using primers GSP4 (Table S5) containing Sal I and ClaI restriction sites to assess the subcellular localization of MaICE1. The PCR product was digested with the above-mentioned enzymes and introduced into the pUC19-GFP vector harboring the GFP reporter gene to generate the fusion construct under the control of the cauliflower mosaic virus $35 \mathrm{~S}$ promoter (CaMV 35S). The fusion construct and the control vector (pUC19-GFP) were separately introduced into Cavendish banana protoplast as previously described [66]. Images were captured from transiently transformed rice protoplast cells grown at $28^{\circ} \mathrm{C}$ using a confocal laser-scanning microscope (LAM510, Carl Zeiss $\mathrm{GmbH}$, Jena, Germany) and analyzed by Image-Pro software.

\section{Generation of transgenic plants by Agrobacterium Tumefaciens-mediated transformation}

The full-length cDNA of MaMAPK3 was subcloned into the pMD18-T vector (TaKaRa, Dalian, China) (Fig. S10A). Plasmid pMD18-T containing MaMAPK3 was amplified using primers (GSP1) harboring SpeI and BamH I restriction sites. The PCR product was digested with these enzymes and introduced into the pCAMBIA 1301-GUS vector (Fig. S10B) to generate the fusion construct 1301-MaMAPK3-GUS under the control of the Ubipromoter. The double-stranded RNA interference (dsRNA) construct (Fig. S10C) was generated via a PCRmediated method using the amplification products from a unique $\mathrm{N}$-terminal region $(300 \mathrm{bp})$ spanning a portion of the $5^{\prime}$-untranslated region and adjacent coding region of the MaMAPK3 gene. The sense strand was then amplified using a primer combination harboring $\mathrm{BamH} \mathrm{I}$ and Hind III restriction sites on the opposed ends of the product, whereas the antisense strand was amplified using a primer combination harboring Pst I and Mul I restriction sites on the opposite ends of the product. These two products were introduced into pYL-RNAi under the control of the Ubi promoter. The newly constructed 1301-MaMAPK3-GUS plant expression vector and RNAi vector were introduced into $A$. tumefaciens strain EHA105 by heat shock [41]. The over-expression vector was adopted for the transformation of 'Dajiao' suspension cultured cells (ECSs), and the RNAi vector was used for the transformation of 'Dajiao' ECSs as previously described [41, 67]. Hygromycin-resistant plants were selected and identified by PCR (HPT and MaICE1) using two pairs of primers (GSP5 and GSP6, Table S5). Only those yielding the expected PCR fragments by both primers were regarded as positive. Moreover, the expression of MaMAPK3 at the mRNA level was examined by qRT-PCR (primer set GSP6, Table S5). The MaACT1 gene (primer set GSP7, Table S5) was selected as a housekeeping gene. Positive banana plants were multiplied vegetatively using meristems of in vitro plantlets. The rooted plantlets were hardened in the greenhouse and used for further analyses.

Specific primers (GSP2) containing Spe I or BamH I restriction sites were used to amplify MaICE1 cDNA. The PCR product was digested with SpeI and BamHI, and then ligated into binary vector $\mathrm{pOx}$ driven by the Zea mays L. polyubiquitin promoter. The constructed binary vector was denoted as pOx-MaICE1. The newly constructed pOx-MaICE1 plant expression vector was introduced into A. tumefaciens strain EHA105 by heat shock [37]. The over-expression vector was adopted for the transformation of Cavendish banana ECSs as previously described (Dou et al., 2016; Hu et al., 2013). Hygromycin-resistant plants were selected and identified by PCR using two pairs of primers (GSP5and GSP8). Only those yielding the expected PCR fragments by both primers were regarded as positive. Moreover, the expression of MaICE1 at the mRNA level was examined by qRT-PCR (primer set GSP9, Table S5). The MaACT1 gene (primer set GSP7) was selected as a housekeeping gene. Positive banana plants were multiplied vegetatively using meristems of in vitro plantlets. The rooted plantlets were hardened in the greenhouse and used for further analyses.

The RNAi construct for MaMPAK3 gene suppression was amplified using the primers GSP10 and GSP11, then the PCR products were cloned into pYLRNAi, which was kindly provided by Dr. Yao-Guang Liu from College of Life Sciences, South China Agricultural University, China. The following procedure was carried out as previously described.

\section{Physiological analyses of MaMAPK3 RNAi transgenic 'Dajiao' plants}

The first young leaf on the top of each of the three plants was collected at each time point $\left(10^{\circ} \mathrm{C}\right.$ for $0,3,5$, and 7 days and recovery after cold stress for 2 days) for each biological replicate. The MDA content was determined by the plant MDA assay kit (Nanjing Jiancheng Bio, Nanjing, China), and the POD activity was tested by the peroxidase assay kit (Nanjing Jiancheng Bio, Nanjing, China). Five replicates were performed for each sample. Three independent experiments were performed as biological replicates.

\section{Cold tolerance assays of the MalCE1 transgenic banana plants}

To avoid interference from plant size and reproduction stage, all plants were chosen from 2-month-old hardened transgenic Cavendish banana, Cavendish banana WT and 'Dajiao' plants, which were grown in plastic pots filled with a mixture of vermiculite and soil (1:1) under a 
photoperiod of $16 \mathrm{~h} / 8 \mathrm{~h}$ (light/dark) at $25^{\circ} \mathrm{C}$. At this stage, there were no apparent differences in plant size and growth between the transgenic and Cavendish banana WT plants. To assess their cold resistance, transgenic lines and WT plants were directly exposed to $10^{\circ} \mathrm{C}$ for $48 \mathrm{~h}$ without pre-acclimation, followed by recovery at an ambient environment for 5 days $[41,42]$. Cold injury and recovery of the plants were recorded and photographed. The degree of cold damage was assessed after recovery as previously described [41].

Two transgenic lines (\#11 and \#13), Cavendish banana WT and 'Dajiao' plants, which were subjected to $10^{\circ} \mathrm{C}$ for $48 \mathrm{~h}$ and subsequent recovery at normal conditions, were adopted in the present study. The leaves were collected before and/or after the cold stress to analyze the electrolyte leakage, Pro content, and MDA level. The leaves used for measurements of electrolyte leakage were cut into $1-\mathrm{cm}$ segments and washed three times with ultrapure water. The segments were placed in tubes containing $5 \mathrm{~mL}$ of ultrapure water and incubated at $25^{\circ} \mathrm{C}$. After $2 \mathrm{~h}$, the electrical conductivity of the bathing solution (L1) was measured. Then the tubes were incubated at $100^{\circ} \mathrm{C}$ for $20 \mathrm{~min}$ and subsequently at $25^{\circ} \mathrm{C}$ for $1 \mathrm{~h}$, and the electrical conductivity (L2) was measured again. The relative electrolyte leakage was calculated by the formula (L1-L0)/(L2-L0) $\times 100$ (L0, the conductivity of ultrapure water) [53]. MDA level and the Pro content were determined using the commercially available kits (Nanjing Jiancheng Bioengineering Institute, China). Five replicates were performed for each sample. Three independent experiments were performed as biological replicates.

\section{RNA-Seq analysis}

Transcriptional profiling of Cavendish banana WT and MaICE1-overexpressing plants (transgenic line \#13) was conducted by RNA-Seq analysis at the Beijing Genomics Institution (BGI). Three biological replicates were adopted for each genotype under normal conditions and cold stress $\left(10^{\circ} \mathrm{C}\right.$ for 1 and $\left.4 \mathrm{~h}\right)$. RNA isolation, library construction, and sequencing on the BGISEQ-500 platform of PE 100 with 30 million reads per sample were performed at BGI (www.genomics.org.cn, BGI, Shenzhen, China). Gene expressions were determined using the RSEM software package [68]. To further confirm the reliability of those transcriptomic data, the expressions of four up-regulated genes were examined by qRT-PCR with specific primers (GSP7, GSP8, GSP9, and GSP10) as shown in Table S5. The DEGseq approach was employed to screen DEGs between groups with the criteria of a fold change $\geq 2$ and adjusted $p$-value $\leq 0.001$ as previously described [69]. Gene Ontology (GO) pathway annotation and enrichment analyses were carried out based on the GO database (http://www.geneontology. org/) and the KEGG pathway database (http://www. genome.jp/kegg/), respectively.

\section{qRT-PCR analysis}

Briefly, $1 \mu \mathrm{g}$ RNA was reversely transcribed into cDNA using ReverTra Ace (Toyobo, Osaka, Japan) with random hexamers. Primers (Table S5) were designed using Primer Premier 5.0 (Premier Biosoft, Palo Alto, USA). PCR was conducted in a $20 \mu \mathrm{L}$ reaction system consisting of $10 \mu \mathrm{L} 2 \times$ SYBR Green PCR Master Mix (Toyobo), $200 \mathrm{nM}$ primers, and $2 \mu \mathrm{L}$ of 1:40-diluted cDNA using the DNA Engine Option 2 Real-Time PCR Detection System and Opticon Monitor software (Bio-Rad, USA). $M a A C T 1$ was selected as a housekeeping gene. The relative expressions of target genes were determined by the $2^{-\Delta \Delta} \mathrm{Ct}$ method [70]. The primers used for qRT-PCR were listed in Table S5.

\section{Phylogenetic tree construction of MAPK}

MAPK genes were isolated from the genome database of Musa (http://banana- genome-hub.southgreen.fr/home) based on annotation and BLAST [71]. Firstly, the sequences belonging to MAPK were downloaded. Then, a further batch of related sequences was obtained from the genome database using Arabidopsis thaliana MAPK genes and the BLAST algorithm (TBLASTN and BLASTP). All of the sequences with the highest similarity were chosen as the candidates. Non-redundant banana MAPK sequences and Arabidopsis thaliana homologs were aligned using the ClustalX program and named according to the phylogenetic tree, which was constructed with FigTree v1.3.1. The deduced amino acid sequences of Arabidopsis thaliana MAPKs were obtained from The Arabidopsis Information Resource (TAIR).

\section{Southern blotting analysis}

Total genomic DNA was isolated from young leaf tissues (3.0 g) of MaMAPK3 RNAi transgenic Dajiao plants and Dajiao WT plants using plant DNA isolation kit (TAKA RA, Japan). Total genomic DNA was isolated from young leaf tissues $(3.0 \mathrm{~g})$ of MaICE1-overexpressing plants and Cavendish banana WT plants using plant DNA isolation kit (TAKARA, Japan). Purified DNA was digested with EcoR V and fractionated on $1.0 \%$ agarose gel. The DNA bands were then transferred onto a nylon membrane (Amersham, USA) by upward capillary in a 20× SSC buffer as previously described [72, 73]. The HPT DNA was adopted as a probe, and it was prepared from Hind III restricted fragment of pYL-RNAi (Clontech) and labeled with digoxigenin. Labeling, hybridization, and washing were carried out using the DIG labeling and Luminescent Detection Kits (Roche, Switzerland). 


\section{Yeast two-hybrids assays}

The Gal-4 reporter-based ProQuest ${ }^{\text {ta }}$ two-hybrid system (Invitrogen, Darmstadt, Germany) was employed to identify the interactions between MAPKs and MAPKKs. Coding sequences (CDSs) of all the MAPK members of Musa were cloned into the prey vector (pDEST32), and CDSs of MAPKKs were introduced into the bait vector (pDEST22). All the combinations of each bait and prey plasmid were PEG-transformed into the yeast strain MaV203 following the protocol of the ProQuest ${ }^{\text {tm }}$ twohybrid system. Positive transformants were first chosen in the synthetic dropout (SD) medium without leucine and tryptophan (SD/-Leu/-Trp), and the culture was transferred to the selection medium without leucine, tryptophan, histidine, and adenine. 3-Amino-1,2,4-triazole (3-AT) was supplemented to the selection plates to inhibit the auto-activation of the prey vectors.

\section{BiFC assay}

The full-length MaPOD P7 and MaICE1 homolog open reading frame was amplified with the following primers set: forward (5'-ggtgagctcggtaccaagctt ATGGCCACCT CCTGGAGAAGCTG-3')/reverse $\quad\left(5^{\prime}\right.$-agcggccgcactagtaagctt GTTCA CCTTCCTGCAATCCAACCT-3') and forward (5'- ggtgagctcggtaccaagctt ATGCTCTCGG GGATCAATGG-3')/reverse $\quad\left(5^{\prime}\right.$-agcggccgcactagtaagcttTGACACT GTATTATCGAAGCCGG-3') respectively, and then introduced into the pMD18-T vector for sequencing. The right MaPOD P7 open reading frame fragment was collected and subcloned into the pRTVnVC vector containing a red fluorescent protein (mCherry) reporter gene (digested with Hind III in advance) to produce the fusion construct Ubi: PODmVenusC under control of the Ubi promoter by using a One Step Cloning Kit (Vazyme Biotech, Nanjing, China). In the same way, the right MaICE1 open reading frame fragment was collected and subcloned into the pRTVnVN vector containing CFP protein reporter gene to produce the fusion construct Ubi: ICE1-mVenusN. The BiFC system used in this study was as described previously with slight modifications [74]. For the interaction studies, protoplasts $(100 \mu \mathrm{l})(1.5-2 \times 106$ cells $)$ were transformed with 5-10 $\mu \mathrm{g}$ of plasmids (Ubi:ICE1mVenusN + Ubi:POD-mVenusC) by the polyethylene glycol (PEG) method with minor modifications [75]. The protoplasts were incubated at $30^{\circ} \mathrm{C}$ for $15 \mathrm{~h}$. The localization or co-localization of mVenus proteins and their markers was assessed with a confocal microscope (Olympus BX61, Tokyo, Japan). The full-length CDS of MaMAPK3 was introduced into pRTVnVC vector, and the full-length CDS of MaICE1 was cloned into pRTVnVN vector. Protoplast isolation and transient expression were carried out as previously described. Empty vectors were co-transformed as negative controls.

\section{Statistical analysis}

A completely randomized design (CRD) was used in the present study. The data were expressed as means \pm standard error (SE). Statistical analysis was carried out using ANOVA by DPS software (version 3.01; Zhejiang University, Hangzhou, China). $P<0.05$ was considered statistically significant.

\section{Limitations}

Limitations include the lack of three independent transgenic lines for gene function analysis and transgenic analysis. In our research, all the transgenic lines showed the same phenotype, so we selected two lines with the most representative and single copy for subsequent experiments such as gene expression and physiological data determination. Three biological duplications were used for each treatment, which made the research results have good repeatability and reliability.

\section{Supplementary Information}

The online version contains supplementary material available at https://doi. org/10.1186/s12870-021-02868-z.

\begin{abstract}
Additional file 1 Fig. S1. (A) Phylogenetic tree of MAPKs from Musa acuminate and Arabidopsis. (B) The expressions of MAPK family in Cavendish banana and 'Dajiao' under 3 h cold stress. Fig. S2. Subcellular localization analysis of MaMAPK3. The ORF of MaMAPK3 was in frame with the GFP C-terminus. (A) Schematic diagrams of the construct used for the subcellular localization assay. (B) Subcellular localization of MaMAPK3 in Cavendish banana protoplast (bar:10 $\mu \mathrm{m})$. Fig. S3. Browning phenomenon of MAMAPK3-overexpressing resistant embryogenic calli. Fig. S4. PCR analysis of MaMAPK3 RNAi transgenic 'Dajiao' lines (M: DNA molecular weight marker; P: plasmid DNA). Fig. S5. Southern blotting analysis of transformed MaMAPK3 RNAi transgenic 'Dajiao' plants. M: DNA molecular weight marker; P: plasmid DNA; WT: wild type. Fig. S6. SubcelIular localization analysis of MaICE1. The ORF of MalCE1 was in frame with the GFP C-terminus. (A) Schematic diagrams of the construct used for the subcellular localization assay. (B) Subcellular localization of MalCE1 in Cavendish banana protoplast (bar:10 $\mu \mathrm{m}$ ). Fig. S7. Generation and molecular identification of transgenic banana plants overexpressing MalCE1. PCR confirmation of the hygromycin-resistant plants using (A) hpt-specific primers or (B) Pubi-MalCE1 primers. M, DNA molecular weight marker; WT, wild-type; -, water; the numbers indicate different transgenic lines (lines $1,5,11$, and 13 are designated as \#1, \#5,\#11, and \#13, respectively); $\mathrm{P}$, plasmid DNA (used as a positive control). (C) Southern blot analysis of MalCE1-overexpressing transgenic 'Cavendish banana' lines. M: DNA Molecular- Weight Marker; P: plasmid DNA; WT: wild type. (D) Expression analysis of MalCE1 in four transgenic lines by RT-PCR. The MaACT1 gene was used as an internal control. Fig. S8. Heatmap of physical interaction verified by $\mathrm{Y} 2 \mathrm{H}$ assay. (A) Physical interaction between MalCE1 and MaMAPKs. (B) Physical interaction between MaMAPKs and MaMKKs. Fig. S9. Heatmap of physical interaction between MaMAPKs and MaMKKs verified by $\mathrm{Y}_{2} \mathrm{H}$ assay. Fig. S10. The plasmid maps of all the constructs used for transgenic in the study.
\end{abstract}

Additional file $\mathbf{2}$ Table S1. Transcriptomic analysis between WT and Ox-ICE1 (line13) under $0 \mathrm{~h}$ cold treatment.

Additional file $\mathbf{3}$ Table S2. Transcriptomic analysis between WT and line13 under $1 \mathrm{~h}$ cold treatment.

Additional file $\mathbf{4}$ Table S3. Transcriptomic analysis between WT and line13 under $4 \mathrm{~h}$ cold treatment.

Additional file $\mathbf{5}$ Table S4. The expression pattern of all identified MAPK cascade genes in the transcriptome. 


\section{Additional file 6 Table S5}

\section{Abbreviations}

WT: wild type; MAPKKK: mitogen-activated protein kinase kinase kinase MAPKK: mitogen-activated protein kinase kinase; MAPK: mitogen-activated protein kinase; bHLH TF: basic-helix-loop-helix transcription factor; qRTPCR: quantitative real-time PCR; GFP: green fluorescent protein; ORF: open reading frame; MDA: malondialdehyde; Pro: proline; DEGs: differentially expressed genes; POD P7: Peroxidase P7; DAB: 3,3'-diaminobenzidine: NBT: Nitroblue Tetrazolium; CaMV 35S: cauliflower mosaic virus 355 promoter; dsRNA: double-stranded RNA interference; ECSs: suspension cultured cells; CDSs: coding sequences; 3-AT: 3-Amino-1,2,4-triazole; PEG: polyethylene glycol; CRD: completely randomized design; SE: standard error

\section{Acknowledgments}

We thank Yaoguang Liu for kindly providing the pYL-RNAi vector.

\section{Authors' contributions}

Q.Y. and G.Y. designed research; J.G., T.D., W.H., C.H., O.S., F. Bi,G.D., H.G., T.D. and C.L., performed research; J.G., T.D. and S.Z. analyzed data; J.G., Q.Y., T.D. and W.H. wrote the paper. All authors have read and approved the manuscript

\section{Funding}

This work was supported by the National Key R\&D Program of China (2018YFD1000302), National Natural Science Foundation of China (31872939), Modern Agricultural Innovation Team Project of Guangdong Province (2018LM2150), R\&D Plan of Guangdong Province Key Fields (2018B020202005), Science and Technology Plan Project of Guangdong Province (2015B070701011), and Guangzhou Scientific Research Plan (201904020033, 201903010010). Thanks to grant No. 2018YFD1000302, No. 31872939 and No. 2018LM2150, we were able to pay for reagents, Thanks to grant No. 2018B020202005 and No. 2015B070701011, we were ableto pay for all kinds of kits, Thanks to grant No. 201904020033and No. 201903010010, we were able to pay forprimers and sequencing. The design of the study and collection, analysis, andinterpretation of data and writing the manuscript were performed by all the authors. The funding bodies just provide the financial support.

\section{Availability of data and materials}

The sequencing raw data of this article have been deposited in a SRA database at the NCBI -https://www.ncbi.nlm.nih.gov/Traces/study/?acc= PRJNA439180.

\section{Ethics approval and consent to participate}

Not applicable.

\section{Consent for publication}

Not applicable.

\section{Competing interests}

The authors declare that they have no competing interests.

\section{Author details}

${ }^{1}$ Institute of Fruit Tree Research, Guangdong Academy of Agricultural Sciences; Key Laboratory of South Subtropical Fruit Biology and Genetic Resource Utilization, Ministry of Agriculture and Rural Affairs, Guangdong Key Laboratory of Tropical and Subtropical Fruit Tree Research, Guangzhou 510640, China. ${ }^{2}$ Guangdong Key Laboratory of Ornamental Plant Germplasm Innovation and Utilization, Environmental Horticulture Research Institute, Guangdong Academy of Agricultural Sciences, Guangzhou 510640, China. ${ }^{3}$ Institute of Biotechnology, Cornell University, Ithaca, NY 14853, USA.
Received: 8 July 2020 Accepted: 1 February 2021

Published online: 17 February 2021

\section{References}

1. D'Hont A, Denoeud F, Aury JM, Baurens FC, Carreel F, Garsmeur O, Noel B, Bocs S, Droc G, Rouard M, et al. The banana (Musa acuminata) genome and the evolution of monocotyledonous plants. NATURE. 2012;488(7410):213-7.

2. Wang Z, Miao H, Liu J, Xu B, Yao X, Xu C, Zhao S, Fang X, Jia C, Wang J et al. Musa balbisiana genome reveals subgenome evolution and functional divergence. NAT PLANTS. 2019

3. Perrier $\mathrm{X}$, De Langhe $\mathrm{E}$, Donohue $\mathrm{M}$, Lentfer C, Vrydaghs L, Bakry F, Carreel F, Hippolyte I, Horry JP, Jenny C, et al. Multidisciplinary perspectives on banana (Musa spp.) domestication. Proc Natl Acad Sci U S A. 2011;108(28): 11311-8.

4. Lescot T. Genetic diversity of banana in figures. FruiTrop. 2008:155:29-33.

5. Paul J, Khanna H, Kleidon J, Hoang P, Geijskes J, Daniells J, Zaplin E, Rosenberg Y, James A, Mlalazi B, et al. Golden bananas in the field: elevated fruit pro-vitamin a from the expression of a single banana transgene. Plant Biotechnol J. 2017;15(4):520-32.

6. Davey MW, Gudimella R, Harikrishna JA, Sin LW, Khalid N, Keulemans J. A draft Musa balbisiana genome sequence for molecular genetics in polyploid, inter- and intra-specific Musa hybrids. BMC Genomics. 2013;14: 683.

7. Langhe $E$, Vrydaghs $L$, de Maret $P$, Perrier $X$, Denham T. Why bananas matter: An introduction to the history of banana domestication. Ethnobot Res Appl. 2008;7.

8. Israeli Y, Lahav E. Injuries to banana caused by adverse climate and weather. Diseases of Banana, Abacá and Enset. 2000:351-79.

9. Chinnusamy V, Zhu J, Zhu JK. Cold stress regulation of gene expression in plants. Trends Plant Sci. 2007;12(10):444-51.

10. Yi J, Lee $Y$, Lee $D$, Cho M, Jeon J, An G. OsMPK6 plays a critical role in cell differentiation during early embryogenesis inOryza sativa. J Exp Bot. 2016: 67(8):2425-37.

11. Lee $Y$, Kim YJ, Kim M, Kwak JM. MAPK cascades in guard cell signal transduction. Front Plant Sci. 2016:7.

12. Kim J, Woo D, Kim S, Lee S, Park H, Seok H, Chung WS, Moon Y. Arabidopsis MKKK20 is involved in osmotic stress response via regulation of MPK6 activity. Plant Cell Rep. 2012;31(1):217-24.

13. Kong $X$, Pan J, Zhang $M$, Xing $X$, Zhou Y, Liu Y, Li D, Li D. ZmMKK4, a novel group C mitogen-activated protein kinase kinase in maize (Zea mays), confers salt and cold tolerance in transgenic Arabidopsis. Plant Cell Environ. 2011:34(8):1291-303.

14. Schweighofer A, Kazanaviciute V, Scheikl E, Teige M, Doczi R, Hirt H, Schwanninger M, Kant M, Schuurink R, Mauch F, et al. The PP2C-type phosphatase AP2C1, which negatively regulates MPK4 and MPK6, modulates innate immunity, jasmonic acid, and ethylene levels in Arabidopsis. Plant Cell. 2007;19(7):2213-24

15. Teige M, Scheikl E, Eulgem T, Dóczi R, Ichimura K, Shinozaki K, Dangl JL, Hirt $H$. The MKK2 pathway mediates cold and salt stress signaling in Arabidopsis. Mol Cell. 2004;15(1):141-52.

16. Zhang M, Su J, Zhang Y, Xu J, Zhang S. Conveying endogenous and exogenous signals: MAPK cascades in plant growth and defense. Curr Opin Plant Biol. 2018:45:1-10

17. Furuya T, Matsuoka D, Nanmori T. Phosphorylation of Arabidopsis thaliana MEKK1 via $\mathrm{Ca}^{2+}$ signaling as a part of the cold stress response. J Plant Res. 2013;126(6):833-40.

18. Yang T, Chaudhuri S, Yang L, Du L, Poovaiah BW. A calcium/Calmodulinregulated member of the receptor-like kinase family confers cold tolerance in plants. J Biol Chem. 2010;285(10):7119-26.

19. Kim SH, Kim HS, Bahk S, An J, Yoo Y, Kim J, Chung WS. Phosphorylation of the transcriptional repressor MYB15 by mitogen-activated protein kinase 6 is required for freezing tolerance in Arabidopsis. Nucleic Acids Res. 2017;45(11):6613-27.

20. Zhao C, Wang P, Wang L, Si T, Hsu C, Zayed O, Yu Z, Zhu J, Zhu Y, Dong J, et al. MAP kinase cascades regulate the cold response by modulating ICE1 protein stability. Dev Cell. 2017:43(5):618-29.

21. Zhang Z, Li J, Li F, Liu H, Yang W, Chong K, Xu Y. OsMAPK3 phosphorylates OsbHLH002/OsICE1 and inhibits its Ubiquitination to activate OsTPP1 and enhances Rice chilling tolerance. Dev Cell. 2017:43(6):731-43.

22. Yu L, Yan J, Yang Y, Zhu W. Overexpression of tomato mitogen-activated protein kinase SIMPK3 in tobacco increases tolerance to low temperature stress. Plant Cell, Tissue and Organ Culture (PCTOC). 2015; 121(1):21-34. 
23. Li H, Ding Y, Shi Y, Zhang X, Zhang S, Gong Z, Yang S. MPK3- and MPK6mediated ICE1 phosphorylation negatively regulates ICE1 stability and freezing tolerance in Arabidopsis. Dev Cell. 2017;43(5):630-42.

24. Li X, Duan X, Jiang H, Sun Y, Tang Y, Yuan Z, Guo J, Liang W, Chen L, Yin J, et al. Genome-wide analysis of basic/helix-loop-helix transcription factor family in rice and Arabidopsis. Plant Physiol. 2006;141(4):1167-84.

25. Wang J, Hu Z, Zhao T, Yang Y, Chen T, Yang M, Yu W, Zhang B. Genomewide analysis of bHLH transcription factor and involvement in the infection by yellow leaf curl virus in tomato (Solanum lycopersicum). BMC Genomics. 2015:16(1):39.

26. Shogo I, Young Hun S, Josephson-Day AR, Miller RJ, Ghislain B, Olmstead RG, Takato I. FLOWERING BHLH transcriptional activators control expression of the photoperiodic flowering regulator CONSTANS in Arabidopsis. Proc Natl Acad Sci U S A. 2012;109(9):3582-7.

27. Tominaga-Wada R, Iwata M, Nukumizu Y, Sano R, Wada T. A full-length Rlike basic-helix-loop-helix transcription factor is required for anthocyanin upregulation whereas the $\mathrm{N}$-terminal region regulates epidermal hair formation. Plant Sci. 2012;183(1):115-22.

28. Bogumil K, Lisa A, Christopher J, Shusei S, Satoshi T, Masayoshi K, Krzysztof S. Conservation of lotus and Arabidopsis basic helix-loop-helix proteins reveals new players in root hair development. Plant Physiol. 2009;151(3): $1175-85$.

29. Ohno S, Hosokawa M, Hoshino A, Kitamura Y, Morita Y, Park Kl, Nakashima A, Deguchi A, Tatsuzawa F, Doi M, et al. A bHLH transcription factor, DvIVS, is involved in regulation of anthocyanin synthesis in dahlia (Dahlia variabilis). J Exp Bot. 2011;62(14):5105-16.

30. Kengo M, Mingzhe Z, Manli Y, Betsy R, Alan L, Rebecca L, Erich G. Participation of the Arabidopsis bHLH factor GL3 in trichome initiation regulatory events. Plant Physiol. 2007;145(3):736-46.

31. Leivar P, Monte E, Oka Y, Liu T, Carle C, Castillon A, Huq E, Quail PH. Multiple phytochrome-interacting bHLH transcription factors repress premature seedling Photomorphogenesis in darkness. Curr Biol. 2008;18(23): 1815-23.

32. Yasuyuki Y, Yasuhisa K, Kaori C, Tadashi Y, Mai O, Sayumi Y, Nobuhiko K, Tomotsugu K, Fumihiko S. Isoquinoline alkaloid biosynthesis is regulated by a unique bHLH-type transcription factor in Coptis japonica. Plant \& Cell Physiology. 2011;52(7):1131-41.

33. Xie XB, Li S, Zhang RF, Zhao J, Chen YC, Zhao Q, Yao YX, You CX, Zhang XS, Hao YJ. The bHLH transcription factor MdbHLH3 promotes anthocyanin accumulation and fruit colouration in response to low temperature in apples. Plant Cell Environ. 2012;35(11):1884-97.

34. Feng $\mathrm{HL}$, Ma NN, Meng X, Zhang S, Wang JR, Chai S, Meng QW. A novel tomato MYC-type ICE1-like transcription factor, SIICE1a, confers cold, osmotic and salt tolerance in transgenic tobacco. Plant Physiol Biochem. 2013;73(73C):309-20.

35. Viswanathan C, Masaru O, Siddhartha K, Byeong-Ha L, Xuhui H, Manu A, Jian-Kang Z. ICE1: a regulator of cold-induced transcriptome and freezing tolerance in Arabidopsis. Genes Dev. 2003;17(8):1043-54.

36. Feng XM. The cold-induced basic helix-loop-helix transcription factor gene MdCIbHLH1 encodes an ICE-like protein in apple. BMC Plant Biol. 2012;12(1):22.

37. Huang XS, Wang W, Zhang Q, Liu JH. A basic helix-loop-helix transcription factor, PtrbHLH, of Poncirus trifoliata confers cold tolerance and modulates peroxidase-mediated scavenging of hydrogen peroxide. Plant Physiol. 2013; 162(2):1178-94.

38. Huang XS, Zhang Q, Zhu D, Fu X, Wang M, Zhang Q, Moriguchi T, Liu JH. ICE1 of Poncirus trifoliata functions in cold tolerance by modulating polyamine levels through interacting with arginine decarboxylase. J Exp Bot. 2015:66(11):3259.

39. Xu W, Zhang N, Jiao Y, Li R, Xiao D, Wang Z. The grapevine basic helix-loophelix (bHLH) transcription factor positively modulates CBF-pathway and confers tolerance to cold-stress in Arabidopsis. Mol Biol Rep. 2014;41(8): 5329-42.

40. Gao J, Zhang S, He W, Shao X, Li C, Wei Y, Deng G, Kuang R, Hu C, Yi G et al. Comparative phosphoproteomics Reveals an Important Role of MKK2 in Banana (Musa spp.) Cold Signal Network. SCI REP-UK. 2017; 7(1):40852.

41. Dou TX, Hu CH, Sun XX, Shao XH, Wu JH, Ding LJ, Gao J, He WD, Biswas MK, Yang QS. MpMYBS3 as a crucial transcription factor of cold signaling confers the cold tolerance of banana. Plant Cell, Tissue and Organ Culture (PCTOC). 2016; 125(1):93-106.

42. Yang Q, Gao J, He W, Dou T, Ding L, Wu J, Li C, Peng X, Zhang S, Yi G. Comparative transcriptomics analysis reveals difference of key gene expression between banana and plantain in response to cold stress. BMC Genomics. 2015;16(1):446.

43. Yang Q, Wu J, Li C, Wei Y, Sheng O, Hu C, Kuang R, Huang Y, Peng X, McCardle JA, et al. Quantitative proteomic analysis reveals that Antioxidation mechanisms contribute to cold tolerance in plantain (Musa paradisiaca L; ABB Group) seedlings. Mol Cell Proteomics. 2012;11(12):185369.

44. Liu K, Hu C, Du F, Zhang YE, Wei Y, Yi G. Over-Expression of the Arabidopsis CBF1 Gene in Dongguandajiao (Musa spp. ABB group) and Detection of Its Cold Resistance. Scientia Agricultura Sinica. 2012.

45. Wood C. Free Radicals in Biology and Medicine. Third Edition. The International Journal of Biochemistry \& Cell Biology. 1999; 31(12):1454.

46. Aslani Aslamarz A, Vahdati K, Hasani D, Rahemi M, Leslie CA. Cold hardiness and its relationship with Proline content in Persian walnut. European Journal of Horticultural Sciences. 2011;76(3):84-90.

47. Aslani Aslamarz A, Vahdati K, Hasani D, Rahemi M, Leslie CA. Supercooling and cold-hardiness of acclimated and Deacclimated buds and stems of Persian walnut cultivars and selections. HortScience horts. 2010;45(11):16627.

48. Ruelland E, Vaultier M, Zachowski A, Hurry V. Chapter 2 Cold Signalling and Cold Acclimation in Plants. In: Advances in Botanical Research., vol. Volume 49: Academic Press; 2009: 35-150.

49. He W, Gao J, Dou T, Shao X, Bi F, Sheng O, Deng G, Li C, Hu C, Liu J, et al. Early cold-induced peroxidases and Aquaporins are associated with high cold tolerance in Dajiao. Front Plant Sci. 2018;9:282.

50. Singh P, Sinha AK. A positive feedback loop governed by SUB1A1 interaction with MITOGEN-ACTIVATED PROTEIN KINASE3 imparts submergence tolerance in Rice. Plant Cell. 2016;28(5):1127-43.

51. Guan Y, Lu J, Xu J, McClure B, Zhang S. Two mitogen-activated protein kinases, MPK3 and MPK6, are required for funicular guidance of pollen tubes in Arabidopsis. Plant Physiol. 2014;165(2):528-33.

52. Zhang $\mathrm{T}$, Chen $\mathrm{S}$, Harmon AC. Protein-protein interactions in plant mitogen-activated protein kinase cascades. J Exp Bot. 2016;67(3):607-18.

53. Zhou M, Li W, Zheng Y, Lin P, Yao X, Lin J. CbRCl35, a cold responsive peroxidase from Capsella bursa-pastoris regulates reactive oxygen species homeostasis and enhances cold tolerance in tobacco. Front Plant Sci. 2016; 7:1599.

54. Huang X, Li K, Jin C, Zhang S. ICE1 of Pyrus ussuriensis functions in cold tolerance by enhancing PUDREBa transcriptional levels through interacting with PuHHP1. SCI REP-UK. 2015;5(1):17620.

55. Zhao C, Lang Z, Zhu J. Cold responsive gene transcription becomes more complex. Trends Plant Sci. 2015;20(8):466-8.

56. Persak H, Pitzschke A. Dominant repression by Arabidopsis transcription factor MYB44 causes oxidative damage and hypersensitivity to abiotic stress. Int J Mol Sci. 2014;15(2):2517-37.

57. Carretero-Paulet L, Galstyan A, Roig-Villanova I, Martínez-García JF, BilbaoCastro JR, Robertson DL. Genome-wide classification and evolutionary analysis of the bHLH family of transcription factors in Arabidopsis, poplar, Rice, Moss, and algae. Plant Physiol. 2010;153(3):1398-412.

58. Liu JH, Peng T, Dai W. Critical cis -acting elements and interacting transcription factors: key players associated with abiotic stress responses in plants. PLANT MOL BIOL REP. 2014;32(2):303-17.

59. Chen $\mathrm{H}$, Hsieh-Feng V, Liao P, Cheng W, Liu L, Yang Y, Lai M, Chang M. The function of OsbHLHO68 is partially redundant with its homolog, AtbHLH112, in the regulation of the salt stress response but has opposite functions to control flowering in Arabidopsis. Plant Mol Biol. 2017;94(4):531-48.

60. Zhao ML, Wang JN, Shan W, Fan JG, Kuang JF, Wu KQ, Li XP, Chen WX, He FY, Chen JY, et al. Induction of jasmonate signalling regulators MaMYC2s and their physical interactions with MalCE1 in methyl jasmonate-induced chilling tolerance in banana fruit. Plant Cell Environ. 2013;36(1):30-51.

61. Yao P, Sun Z, Li C, Zhao X, Li M, Deng R, Huang Y, Zhao H, Chen H, Wu Q Overexpression of Fagopyrum tataricum FtbHLH2 enhances tolerance to cold stress in transgenic Arabidopsis. Plant Physiol Biochem. 2018;125:85-94.

62. Ning T, Hua Z, Xianghua L, Jinghua X, Lizhong X. Constitutive activation of transcription factor OsbZIP46 improves drought tolerance in rice. Plant Physiol. 2012;158(4):1755-68.

63. Ding Y, Li H, Zhang X, Xie Q, Gong Z, Yang S. OST1 kinase modulates freezing tolerance by enhancing ICE1 stability in Arabidopsis. Dev Cell. 2015; 32(3):278-89,

64. Thompson JD, Higgins DG, Gibson TJ. CLUSTAL W: improving the sensitivity of progressive multiple sequence alignment through sequence weighting, 
position-specific gap penalties and weight matrix choice. Nucleic Acids Res. 1994;22(22):4673-80.

65. Kumar S, Stecher G, Li M, Knyaz C, Tamura K. MEGA X: Molecular evolutionary genetics analysis across computing platforms. Mol Biol Evol. 2018;35(6):1547-9.

66. Moin M, Bakshi A, Madhav MS, Kirti PB. Cas9/sgRNA-based genome editing and other reverse genetic approaches for functional genomic studies in rice. BRIEF FUNCT GENOMICS. 2018;17(5):339-51.

67. Hu C, Wei Y, Huang Y, Yi G. An efficient protocol for the production of chit42 transgenic Furenzhi banana (Musa spp. AA group) resistant to Fusarium oxysporum. In Vitro Cellular \& Developmental Biology - Plant. 2013;49(5):584-92.

68. Li B, Dewey CN. RSEM: accurate transcript quantification from RNA-Seq data with or without a reference genome. BMC BIOINFORMATICS. 2011;12(1):323.

69. Huang C, Zhao F, Lin Y, Zheng S, Liang S. Han S. RNA-Seq analysis of global transcriptomic changes suggests a roles for the MAPK pathway and carbon metabolism in cell wall maintenance in a Saccharomyces cerevisiae FKS1 mutant. Biochemical \& Biophysical Research Communications. 2018;500(3): 603-8.

70. Livak KJ, Schmittgen TD. Analysis of relative gene expression data using real-time quantitative PCR and the 2(-Delta Delta $C(T))$ method. METHODS. 2001:25(4):402-8.

71. Martin G, Baurens FC, Droc G, Rouard M, Cenci A, Kilian A, Hastie A, Doležel J, Aury JM, Alberti A. Improvement of the banana "Musa acuminata" reference sequence using NGS data and semi-automated bioinformatics methods. BMC Genomics. 2016;17(1):243.

72. Matheka J, Tripathi JN, Merga I, Gebre E, Tripathi L. A simple and rapid protocol for the genetic transformation of Ensete ventricosum. Plant Methods. 2019;15:130.

73. Southern EM. Detection of specific sequences among DNA fragments separated by gel electrophoresis. J Mol Biol. 1975;98(3):503 N3, 508-509, N5, 517.

74. Wamaitha MJ, Yamamoto R, Wong HL, Kawasaki T, Kawano Y, Shimamoto K. OsRap2.6 transcription factor contributes to rice innate immunity through its interaction with Receptor for Activated Kinase-C 1 (RACK1). RICE. 2012; 5(1):35.

75. Marx JL. Rice plants regenerated from protoplasts: the ability to regenerate rice protoplasts means that for the first time a major cereal will become subject to modern biotechnological methods. SCIENCE. 1987;235(4784):31-2.

\section{Publisher's Note}

Springer Nature remains neutral with regard to jurisdictional claims in published maps and institutional affiliations.

Ready to submit your research? Choose BMC and benefit from:

- fast, convenient online submission

- thorough peer review by experienced researchers in your field

- rapid publication on acceptance

- support for research data, including large and complex data types

- gold Open Access which fosters wider collaboration and increased citations

- maximum visibility for your research: over $100 \mathrm{M}$ website views per year

At BMC, research is always in progress.

Learn more biomedcentral.com/submissions 